\title{
A novel method to study the phononic crystals with fluid structure interaction and hybrid uncertainty
}

\author{
X. Y. $\operatorname{Lin}^{1}$, Eric Li ${ }^{2}$, Z. C. $\mathrm{He}^{1,},{ }^{*}, \mathrm{Y} . \mathrm{Wu}^{1}$ \\ ${ }^{I}$ State Key Laboratory of Advanced Design and Manufacturing for Vehicle Body, Hunan University, \\ Changsha, 410082 P. R. China \\ ${ }^{2}$ School of Science, Engineering \& Design, Teesside University, Middlesbrough, UK
}

\begin{abstract}
Traditional finite element methods for the phononic crystals (PCs) with fluid-structure interaction (FSI) are generally based on the deterministic methodology. However, the uncertainty between the design and the manufactural values of the PCs and the uncertainty from the material properties deviation are always unavoidable, which may give rise to the discrepancy of the physical responses. This paper presents a hybrid uncertain mass-redistributed finite element method (HUMR-FEM) to quantitatively describe the uncertainty of the PCs. The MR-FEM is proposed to deal the PCs with FSI to minimize dispersion error, and the hybrid uncertainty is treated as the random uncertainty type with bounded distribution parameters instead of a precise one. The uncertainty model can be transformed into the deterministic computation of the extreme bounds of the statistical characteristics. The influences of the hybrid uncertainty on the physical responses including the band structures and mode shape of the PCs with FSI are investigated. The accuracy and efficiency of the proposed method are validated through several numerical examples including different materials combinations and lattice forms.
\end{abstract}

Key words: Interval random uncertainty; Extreme bounds; Fluid structure interaction; Band structures

\footnotetext{
* Corresponding author

E-mail address: hezhicheng815@hnu.edu.cn (Z.C. He)
} 


\section{Introduction}

Recent three decades have witnessed the development of two categories of artificial structure materials used to manipulate the acoustic and elastic waves propagation, they are known as the phononic crystals (PCs) and the acoustic metamaterials (AMs) [1-3]. An important property of the PCs and AMs is that they can induce bandgaps where all propagating Bloch waves are prohibited within a certain frequency range, resulting in a ground-breaking phenomenon, such as negative refraction [4,5], imaging with sub-wavelength [6,7], and acoustic cloaking [8,9]. A deep investigation of the bandgaps will significantly help us understand wave propagation, reveal new physical phenomena, and design novel acoustic/elastic wave materials $[10,11]$.

So far, traditional theoretical studies for the bandgaps of the PCs are generally based on the deterministic methodology. However, the deviations between the actual and the ideal PCs due to the manufacture error, materials defects and geometry sizes are always unavoidable, which may result in the uncertainty in the physical responses [12-15]. Sukhovich et al. [16] found that the losses or the imperfections of the crystal may lead to a $10 \%$ deviation for the resolution quality during the experiment of the focusing, which is inevitable in some practical system. In the experiment designed to obtain high-quality focus imaging, Zhang et al. [17] observed that disagreement of refractive index between the experimental and the exact solution is attributed to the mismatching between the designed value and the manufactural value of the AMs lumped network; besides, they also found that a little material loss during the 
measurement can dramatically reduce the focusing resolution. These studies indicate that a complex AMs and PCs system usually contain many uncertain factors which cannot be ignored. Quantifying these uncertainties evokes a series of research interests [18]. Li et al.[14] predicted the bounds of physical responses in the PCs with the interval uncertainty. He et al. [15] quantified the random uncertainty of the solid-solid PCs, meanwhile, the uncertain variables are assumed to obey random distribution with known expectations and standard variances. These works demonstrate that the band structures and the mode shapes of the PCs and AMs are strongly affected by the uncertain parameters. However, quantifying the impact of the uncertainties on the physic responses of the PCs involving FSI remains two challenges. The first challenge is the treatment of the uncertainties, and the other one is the development of efficient and accurate numerical methods to handle the fluid structure interaction issues in the PCs.

In the treatment for the uncertainties of the PCs, establishing a practical model to accurately reflect the uncertainty in the PCs is very significant. The uncertain problems may transform into either the non-probabilistic model [14] or the probabilistic model $[13,19]$. The latter can be applied to treat the uncertain problems with priori statistics information, but the computational effort is a great burden since a lot of samples of the uncertain parameters is required. Besides, during the calculation of the band structures and the mode shapes, the Floquet-Bloch periodicity is applied along the boundaries of a single unit cell and is different with the traditional systems $[10,13]$. In this case, it may be unreasonable to regard the representative unit cell as a 
pure random model with the identical distribution parameters as all the other unit cells are considered to be the same as the representative one. The interval model may be the most frequently used uncertain model to describe the uncertainty emerged in the PCs, such as $[14,20,18]$. However, the results from this model may be ultra-conservative as the computation only considers the known extreme bounds.

Except for the pure random model and the interval model, the hybrid uncertain model [21,22] is constructed to integrate the advantages of both the non-probabilistic and the probabilistic model, since a number of PCs actually involve both types of uncertainties in the design [18]. Generally, there are two kinds of hybrid uncertain model including the hybrid random and interval model and the interval random model. In the former one, the random variables and interval variables coexist, where a part of variables with precise statistical information are classified as random model and the rest part of variables with extreme bounds belong to the interval model. However, the sufficient information of the random variables the hybrid random and interval uncertainty is still required, which means this hybrid case is slightly simplified from the random model and it may still require huge computational cost. In this case, the interval random model with a moderate computational cost is more preferred as only the distribution type of the random and the extreme bounds of the stochastics characteristics are needed. In this model, the uncertainty variables are considered as random type whose statistical parameters locate in an interval range. Besides, the PCs system involving interval random uncertainty is still at its preliminary phase, the effects of the hybrid uncertainty on the physical responses remain unresolved. 
Therefore, our research interest will focus on this interval random uncertain model.

In the second challenge, the development of reliable and accurate numerical methods is of great significance for the study of wave propagation in different types of PCs [23-27]. The finite element method (FEM) [28-31] is known very effective to deal with complex boundaries and interface and thus is frequently used in the simulation of the PCs and AMs. However, in the analysis of PCs involving FSI the longitudinal and shear waves propagate simultaneously in the solids, while only longitudinal wave exists in the ideal fluids. In this case, the computational efficiency and accuracy of traditional FEM may be low due to the complicated FSI and the large acoustic impedance mismatch at the boundary [32-35]. Besides, as the traditional FEM is applied to the numerical simulation of the acoustic domain, the discretization of the standard weak formulations will produce evident accuracy loss compared with the continuous equations. Specifically, based on the traditional FEM the velocity of the longitudinal wave used in the simulation is faster than the real velocity of the acoustic material and the dispersion error is thus inevitably induced with the “overly-stiff” characteristic [34,36].

An efficient strategy to minimize this error is the application of the matching technique between the discretized system [31,37]. Recently, the mass-redistributed finite element method (MR-FEM) \{He, 2016 \#220;Li, 2016 \#932;Li, 2016 \#939;Li, $2016 \# 946 ; \mathrm{Li}, 2017 \# 1038$ \}, which balances the mass and stiffness matrices of a discretized model based on flexible integration points, has been proven to be effective in reducing the dispersion error in the fluid domain. With the employment of standard 
triangular elements, the balance operation is simply accomplished through only shifting the integration points in the assembling process of the mass matrix, thus the corresponding changing from the standard FEM code is almost negligible, which makes the MR-FEM much more efficient in the simulation of the acoustic wave. In the solid domain the coupled longitudinal wave and shear wave propagate simultaneously, the lumped mass matrix is served to study the propagation of elastic wave in the solid domain, leading to a compromise between the longitudinal wave and the shear wave [34], providing a desirable result for the simulation of the elastic waves.

Based on the above two purposes, we will propose an effective, efficient and robust method, namely the hybrid uncertain MR-FEM (HUMR-FEM), to deal with the hybrid uncertain PCs involving FSI. To our best knowledge, the combination of the interval random uncertainty and the MR-FEM would be the first trial. The paper is organized as follows. In Section 2, we formulate the MR-FEM framework to accurately study the physical responses of the PCs considering FSI. In Section 3, based on the MR-FEM, the hybrid uncertainty analysis is given to quantify the impact of uncertainties. The effectiveness and the computational efficiency of HUMR-FEM are validated through some examples in Section 4 and the conclusions are draw in Section 5 .

\section{MR-FEM solution for fluid/solid and solid/fluid PCs}

\subsection{Wave propagation in fluid/solid and solid/fluid PCs}

In this subsection, a unit cell of $2 \mathrm{D}$ periodic PCs considering the fluid structure 
interaction shown in Fig. 1 is introduced. The governing motion equations for the in-plan elastic waves in an isotropic solid $\Omega_{s}$ can be written as:

$$
\nabla \cdot\left[\mu \nabla u_{i}(\mathbf{r})\right]+\nabla \cdot\left(\mu \frac{\partial u_{i}(\mathbf{r})}{\partial x_{i}}\right)+\frac{\partial}{\partial x_{i}}\left[\lambda \nabla \cdot u_{i}(\mathbf{r})\right]=\rho_{s} \frac{\partial^{2} u_{i}(\mathbf{r})}{\partial t^{2}}, i=1,2
$$

where $\nabla=(\partial / \partial x, \partial / \partial y)$ is the gradient operator, $\mathbf{u}(\mathbf{r})=\left(u_{x}, u_{y}\right)^{\mathrm{T}}$ is the displacement vector at the position $\mathbf{r}=(x, y)$, the subscript index $s$ denotes the parameter of the solid domain, $\rho_{s}$ is the mass density; $\lambda$ and $\mu$ are the Lamé's first constant and the shear modulus, which can be obtained as follows:

$$
\lambda=\frac{v E}{(1+v)(1-2 v)}, \quad \mu=\frac{E}{2(1+v)}
$$

where $E$ denotes the Young's modulus and $v$ represents Poisson's ratio of the material. In the solid domain the constitutive relationship can be expressed as $\boldsymbol{\sigma}=\mathbf{D} \boldsymbol{\varepsilon}$, where $\boldsymbol{\sigma}$ denotes the stress vector, $\boldsymbol{\varepsilon}$ denotes the strain vector; $\mathbf{D}$ is the elastic matrix, and they are given as follows:

$$
\boldsymbol{\sigma}=\left[\begin{array}{c}
\sigma_{x x} \\
\sigma_{y y} \\
\tau_{x y}
\end{array}\right], \mathbf{D}=\left[\begin{array}{ccc}
\lambda+2 \mu & \lambda & 0 \\
\lambda & \lambda+2 \mu & 0 \\
0 & 0 & \mu
\end{array}\right], \boldsymbol{\varepsilon}=\left[\begin{array}{c}
\varepsilon_{x x} \\
\varepsilon_{y y} \\
\gamma_{x y}
\end{array}\right]
$$

where $\varepsilon_{x x}=\partial u_{x} / \partial x, \varepsilon_{y y}=\partial u_{y} / \partial y, \gamma_{x y}=1 / 2\left(\partial u_{y} / \partial x+\partial u_{x} / \partial y\right)$. Then, Eq. (1) can be respectively rewritten in $x$ and $y$ direction [32]:

$$
\begin{aligned}
& (\lambda+2 \mu) \frac{\partial^{2} u_{x}}{\partial x^{2}}+(\lambda+\mu) \frac{\partial^{2} u_{y}}{\partial x \partial y}+\mu \frac{\partial^{2} u_{x}}{\partial y^{2}}=\rho_{s} \frac{\partial u_{x}^{2}}{\partial t^{2}} \\
& \mu \frac{\partial^{2} u_{y}}{\partial x^{2}}+(\lambda+\mu) \frac{\partial^{2} u_{x}}{\partial x \partial y}+(\lambda+2 \mu) \frac{\partial^{2} u_{y}}{\partial y^{2}}=\rho_{s} \frac{\partial u_{y}^{2}}{\partial t^{2}}
\end{aligned}
$$

Analogically, the governing equations for the acoustic wave in an ideal fluid $\Omega_{f}$ can be expressed as: 


$$
\frac{1}{\rho_{f}} \nabla\left(\nabla p_{t}\right)-\frac{1}{\kappa} \frac{\partial p_{t}^{2}}{\partial t^{2}}=0, \text { in } \Omega_{f}
$$

where $\kappa=\rho_{f} c^{2}$ is the bulk modulus, $c$ is the wave velocity of fluid domain, $\rho_{f}$ is the mass density and $p_{t}$ denotes the time-harmonic acoustic wave with the form $p=p_{t} e^{j \omega t}$ (where $\omega$ is the angular frequency), where $p$ represents complex acoustic pressure subjected to the Helmholtz equation: $\Delta p+k^{2} p=0$, where $k=\omega / c$.
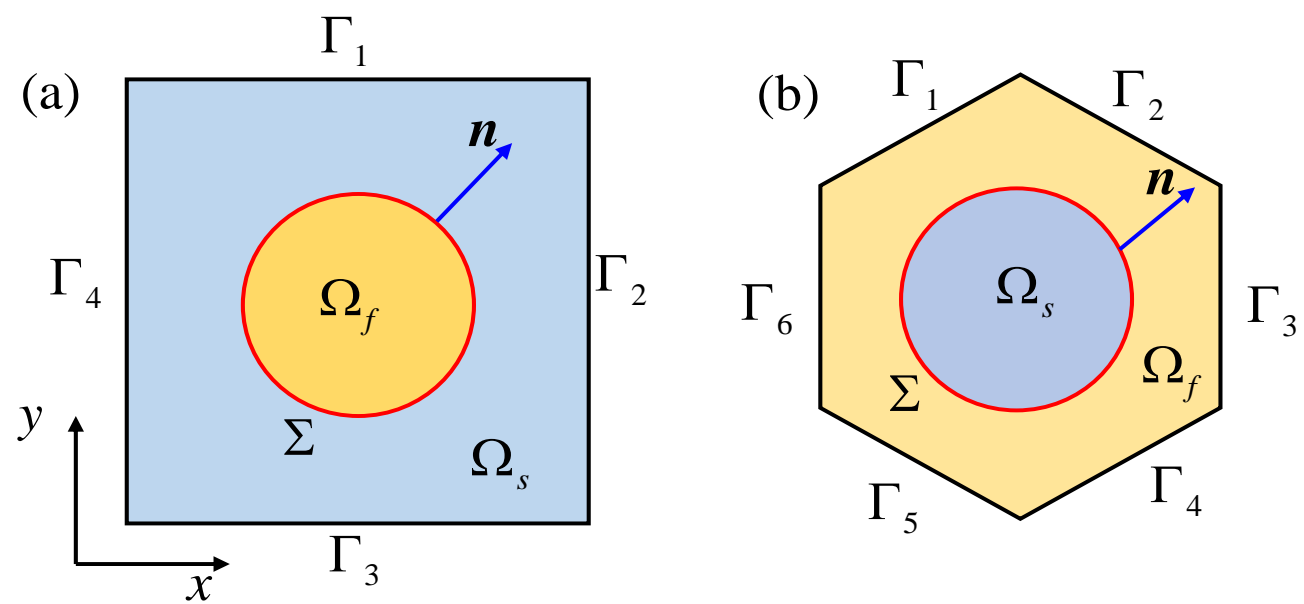

Fig. 1 A representative unit cell of 2D periodic PCs considering the fluid structure interaction (a) Square lattice; (b) Hexangular lattice

\subsection{MR-FEM solution for fluid/solid and solid/fluid PCs}

For effective discussion, the discretization using standard FEM is firstly briefed..

\subsubsection{Variational formulation}

As shown in Fig. 1, the whole domain is divided into two parts including solid part $\Omega_{s}$ and fluid part $\Omega_{f}$, and they are coupled through the interface $\Sigma$. The standard Galerkin approach for solid in a 2D structure can be written as [34]:

$$
\mathbf{M}_{s} \ddot{\mathbf{u}}+\mathbf{K}_{s} \mathbf{u}=\mathbf{F}_{f \rightarrow s}+\mathbf{F}_{s}
$$

where $\mathbf{u}$ is the displacement vector; the subscript index " $s$ " denotes the quantity in the solid domain. $\mathbf{F}_{s}$ denotes external body force acting on the solid; $\mathbf{F}_{f \rightarrow s}$ is the coupled 
force vector from the fluid through the FSI boundary $\Sigma ; \mathbf{K}_{s}$ and $\mathbf{M}_{s}$ are the global stiffness and mass matrices, respectively, and are defined as follows:

$$
\mathbf{K}_{s}=\int_{\Omega_{s}} \mathbf{B}_{s}^{\mathrm{T}} \mathbf{D} \mathbf{B}_{s} \mathrm{~d} \Omega, \quad \mathbf{M}_{s}=\rho_{s} \int_{\Omega_{s}} \mathbf{N}_{s}^{\mathrm{T}} \mathbf{N}_{s} \mathrm{~d} \Omega
$$

where $\mathbf{N}_{s}$ is the Lagrange shape function, $\mathbf{B}_{s}=\nabla \mathbf{N}_{s}$ is the strain matrix.

Similarly, the acoustic wave Eq. (5) can be rewritten as:

$$
\mathbf{M}_{f} \ddot{\mathbf{p}}+\mathbf{K}_{f} \mathbf{p}=\mathbf{F}_{s \rightarrow f}+\mathbf{F}_{f}
$$

where the subscript index " $f$ " denotes the quantity in the fluid domain. $\mathbf{F}_{f}$ is the external pressure vector, $\mathbf{F}_{s \rightarrow f}$ denotes the coupled force vector on the FSI boundary $\Sigma, \mathbf{p}$ is the pressure, $\mathbf{K}_{f}$ and $\mathbf{M}_{f}$ are the global stiffness and mass matrices, respectively, and they are defined as:

$$
\mathbf{K}_{f}=\int_{\Omega_{f}} \mathbf{B}_{f}^{\mathrm{T}} \mathbf{B}_{f} \mathrm{~d} \Omega, \mathbf{M}_{f}=\frac{1}{c^{2}} \int_{\Omega_{f}} \mathbf{N}_{f}^{\mathrm{T}} \mathbf{N}_{f} \mathrm{~d} \Omega
$$

where $\mathbf{B}_{f}=\nabla \mathbf{N}_{f}$ is the gradient of the Lagrange shape function $\mathbf{N}_{f}$.

To determine the coupled forces $\mathbf{F}_{f \rightarrow s}$ and $\mathbf{F}_{s \rightarrow f}$, the displacement and stress continuity conditions between the fluid and solid at the interface $\Sigma$ should be considered, which are described as:

Displacement continuity

Normal stress continuity

$$
\begin{gathered}
u \cdot \mathbf{n}=u_{f} \cdot \mathbf{n} \text { on } \Sigma \\
\sigma \cdot \mathbf{n}=p \cdot \mathbf{n} \text { on } \Sigma
\end{gathered}
$$

where $u$ and $u_{f}$ are the displacements of the solid and fluid domains along the coupled interface, respectively; $\mathbf{n}=\left(n_{x}, n_{y}\right)^{\mathrm{T}}$ is the normal vectors external to the interface $\Sigma$. Using the equilibrium conditions of the tractions in the direction perpendicular to the interface yields: 


$$
\sigma_{x x} n_{x}+\tau_{x y} n_{y}=p n_{x}, \quad \tau_{x y} n_{x}+\sigma_{y y} n_{y}=p n_{y}
$$

The excitation generated from the solid domain can be considered as the velocity input (velocity boundary) exerting on the fluid [38]:

$$
\mathbf{F}_{s \rightarrow f}=\oint_{\Sigma}-j \rho_{f} \omega \mathbf{N}_{f}^{\mathrm{T}} v \cdot \mathbf{n} \mathrm{d} \Sigma
$$

where the integration of the force is along the closed boundary and $v \cdot \mathbf{n}$ denotes the normal velocity input. Since the displacement continuity is applied on the interface, we can arrive at $v \cdot \mathbf{n}=\dot{u} \cdot \mathbf{n}$, thus the coupling excitation $\mathbf{F}_{s \rightarrow f}$ can be described as:

$$
\begin{aligned}
\mathbf{F}_{s \rightarrow f} & =\oint_{\Sigma}-j \rho_{f} \omega \mathbf{N}_{f}^{\mathrm{T}} \dot{u} \cdot \mathbf{n} \mathrm{d} \Sigma=-\rho_{f} \oint_{\Sigma} \mathbf{N}_{f}^{\mathrm{T}} j \omega \dot{u} \cdot \mathbf{n} \mathrm{d} \Sigma \\
& =-\rho_{f}\left(\oint_{\Sigma} \mathbf{N}_{f}^{\mathrm{T}} \ddot{u} \cdot \mathbf{n} \mathrm{d} \Sigma\right)=-\rho_{f}\left(\oint_{\Sigma} \mathbf{N}_{f}^{\mathrm{T}} \mathbf{n} \mathbf{N}_{s} \mathrm{~d} \Sigma\right) \ddot{\mathbf{u}}
\end{aligned}
$$

According to the normal stress continuity, the fluid force acting on the solid domain can be calculated by:

$$
\mathbf{F}_{f \rightarrow s}=-\oint_{\Sigma} \mathbf{N}_{s}^{\mathrm{T}} \tau \mathrm{d} \Sigma=-\oint_{\Sigma} \mathbf{N}_{s}^{\mathrm{T}} p \cdot \mathbf{n} \mathrm{d} \Sigma=-\left(\oint_{\Sigma} \mathbf{N}_{s}^{\mathrm{T}} \mathbf{n} \mathbf{N}_{f} \mathrm{~d} \Sigma\right) \mathbf{p}
$$

It should be emphasized that in the fluid domain, $\sigma_{x x}=\sigma_{y y}=-p$ and $\sigma_{x y}=0$ are satisfied.

The spatial coupled matrix between the fluid and structure is thus introduced:

$$
\mathbf{H}=\oint_{\Sigma} \mathbf{N}_{s}^{\mathrm{T}} \mathbf{n} \mathbf{N}_{f} \mathrm{~d} \Sigma
$$

Using Eq. (14) and combining Eqs. (6) (8) and (12) yield an unsymmetrical equation of the interaction system:

$$
\left[\begin{array}{cc}
\mathbf{M}_{s} & 0 \\
\rho_{f} \mathbf{H}^{\mathrm{T}} & \mathbf{M}_{f}
\end{array}\right]\left\{\begin{array}{l}
\ddot{\mathbf{u}} \\
\ddot{\mathbf{p}}
\end{array}\right\}+\left[\begin{array}{cc}
\mathbf{K}_{s} & -\mathbf{H} \\
0 & \mathbf{K}_{f}
\end{array}\right]\left\{\begin{array}{l}
\mathbf{u} \\
\mathbf{p}
\end{array}\right\}=\left[\begin{array}{l}
\mathbf{F}_{s} \\
\mathbf{F}_{f}
\end{array}\right]
$$

For the simplicity, the following notations are used:

$$
\mathbf{M}=\left[\begin{array}{cc}
\mathbf{M}_{s} & 0 \\
\rho_{f} \mathbf{H}^{\mathrm{T}} & \mathbf{M}_{f}
\end{array}\right], \mathbf{K}=\left[\begin{array}{cc}
\mathbf{K}_{s} & -\mathbf{H} \\
0 & \mathbf{K}_{f}
\end{array}\right], \quad \mathbf{U}=\left[\begin{array}{l}
\mathbf{u} \\
\mathbf{p}
\end{array}\right], \quad \mathbf{F}=\left[\begin{array}{c}
\mathbf{F}_{s} \\
\mathbf{F}_{f}
\end{array}\right]
$$


where $\mathbf{M}$ and $\mathbf{K}$ are the global stiffness and mass matrices, $\mathbf{U}$ and $\mathbf{F}$ are the global response and the force vectors, respectively.

Assuming that the external excitation is time harmonic, the dynamic equilibrium equation of the fluid/solid and solid /fluid PCs can be expressed by:

$$
\left[\mathbf{K}-\omega^{2} \mathbf{M}\right] \mathbf{U}=\mathbf{F}
$$

\subsubsection{MR-FEM formulation}

In the traditional FEM, the linear triangular elements are used for discretization, where the stiffness matrix of the fluid domain in Eq. (9) is assembled without the numerical integration, i.e., the stiffness matrix of an element is determined by the product of the integrand and the area of the integration domain. Besides, as the integral function of the mass matrix is no longer a constant, Eq. (9) can be calculated either through the analytically (area coordinating properties [39]) or the numerically ways (conventional Gauss integral approach). Currently, two kinds of Gauss integration points corresponding to the lumped matrix and the consistent matrix are mostly used to assemble the mass matrix. However, using these two kinds of matrices yields a same dispersion error of second order and they cannot balance perfectly with the stiffness matrix and further reduce the dispersion error [36]. To this end, the MR-FEM is proposed to find the best locations of the integration points for the fluid mass matrix.

In the discretization of the mass matrix, three flexible integration points $Q, S$ and $R$ (as shown in Fig. 2) in the triangular elements are used: 


$$
\begin{aligned}
& \text { Point } Q: x_{i}=\frac{1-\alpha}{2}, \quad y_{i}=\frac{1-\alpha}{2} ; \\
& \text { Point } S: x_{i}=\alpha, \quad y_{i}=\frac{1-\alpha}{2} ; \\
& \text { Point } R: x_{i}=\frac{1-\alpha}{2}, \quad y_{i}=\alpha
\end{aligned}
$$

where $\alpha \in[0,1]$ denotes the different integrations, e.g. $\alpha=1$ represents the lumped mass matrix and $\alpha=2 / 3$ represents the consistent mass matrix; $x_{i}$ and $y_{i}$ are the coordinates of the integration points.

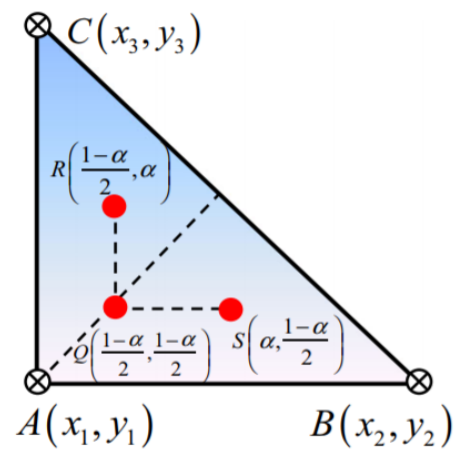

(a) $\alpha \in(0,1)$, MR-FEM

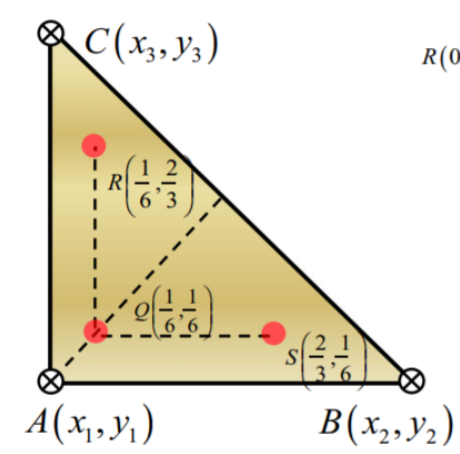

(b) $\alpha=2 / 3$,

Consistent mass matrix

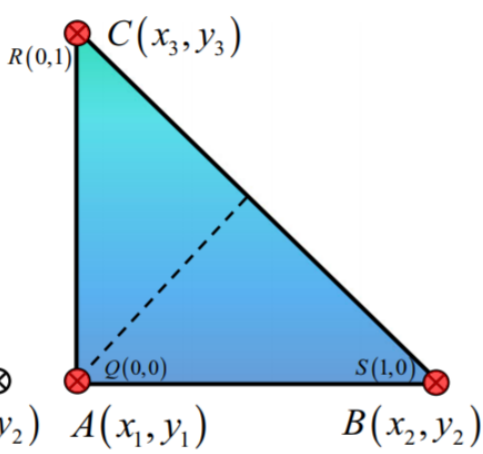

(c) $\alpha=1$, Lumped mass matrix

$\otimes$ Nodes of filed $(A, B, C) \bigcirc$ Integration point $(Q, S, R)$

Fig. 2: The basic framework of MR-FEM

In the fluid domain, the mass matrix of each element can be expressed with a parameter $\alpha$ which is related to the locations of the integration points:

$$
\mathbf{M}_{f}^{e}=\frac{1}{3 c^{2}} A^{e}\left[\begin{array}{ccc}
\alpha^{2}+\frac{(1-\alpha)^{2}}{2} & \alpha(1-\alpha)+\frac{(1-\alpha)^{2}}{4} & \alpha(1-\alpha)+\frac{(1-\alpha)^{2}}{4} \\
\alpha(1-\alpha)+\frac{(1-\alpha)^{2}}{4} & \alpha^{2}+\frac{(1-\alpha)^{2}}{2} & \alpha(1-\alpha)+\frac{(1-\alpha)^{2}}{4} \\
\alpha(1-\alpha)+\frac{(1-\alpha)^{2}}{4} & \alpha(1-\alpha)+\frac{(1-\alpha)^{2}}{4} & \alpha^{2}+\frac{(1-\alpha)^{2}}{2}
\end{array}\right]
$$

where $\mathbf{M}_{f}^{e}$ and $A^{e}$ are the mass matrix and the area of each triangular element, respectively. By using flexible integration points, the actual wavenumber $k_{e}$ can be expressed by the approximate wavenumber $k_{a}$ as [34]: 


$$
\left(k_{e}\right)^{2}=\frac{1}{d^{2}} \frac{24-12\left[\cos \left(k_{1} d\right)+\cos \left(k_{2} d\right)\right]}{3\left(1-2 \alpha+3 \alpha^{2}\right)+\left(1+2 \alpha-3 \alpha^{2}\right)\left[\cos \left(k_{1} d\right)+\cos \left(k_{2} d\right)+\cos \left(k_{1} d+k_{2} d\right)\right]}
$$

where $d$ is the element size representing the length of the shortest edge in the mesh, $k_{1}=k_{a} \cos \theta, k_{2}=k_{a} \sin \theta$ with $\theta$ denoting the wave propagation angle with respect to the normal.

Eq. (20a) shows that the actual wavenumber $k_{e}$ is a function of $\alpha$ related to the location of the integration point. As the pressure field in the fluid domain without considering the temporally harmonic item $e^{i \omega t}$ is expressed as $p(\mathbf{x})=P e^{i\left(k_{a} \cos \theta x+k_{a} \sin \theta y\right)}$, where $P$ denotes the pressure amplitude and $\mathbf{x}$ stands for the position. Generally, $k_{a}$ representing the approximated wavenumber is used in the standard FEM. However, $k_{a}$ is different from the actual number $k_{e}$. Applying the Taylor expansion for the right hand of Eq. (20a) yields the difference between these two wavenumbers:

$$
k_{e}=k_{a}-\frac{d^{2}\left(k_{a}\right)^{3}}{24} \cdot\left[\begin{array}{l}
2 \cos ^{2} \theta\left(\cos ^{2} \theta-1\right)- \\
\left(1+2 \alpha-3 \alpha^{2}\right) \sin 2 \theta-\left(1+4 \alpha-6 \alpha^{2}\right)
\end{array}\right]+o\left(k_{a}\right)^{5}
$$

The relative dispersion error $e(\alpha, k)$ is defined as:

$$
e(\alpha, k)=\left|\frac{k_{e}-k_{a}}{k_{a}}\right|=\left|\frac{d^{2}\left(k_{a}\right)^{2}}{24} \cdot\left[\begin{array}{l}
2 \cos ^{2} \theta\left(\cos ^{2} \theta-1\right)- \\
\left(1+2 \alpha-3 \alpha^{2}\right) \sin 2 \theta-\left(1+4 \alpha-6 \alpha^{2}\right)
\end{array}\right]+o\left(k_{a}\right)^{4}\right|
$$

where $k_{e}$ and $k_{a}$ are the actual and the approximate wavenumbers, respectively. It should be noted that, to obtain a minimal dispersion error the parameter $\alpha$ is to be determined. Through some rigorous derivation that aims to minimize the relative dispersion error, 
we have found that the optimal value of $\alpha$ is: $\alpha=(2 \pm \sqrt{10}) / 6$, the detailed derivations can be found in our previous work [34].

In the solid domain, the balancing between the mass and stiffness matrices is much more complicated since the in-plane longitudinal wave and shear wave are coupled. The wavelength ratio between the longitudinal and the shear waves are expressed by:

$$
\frac{\ell_{p}}{\ell_{s}}=\sqrt{\frac{(1+v)}{(1-2 v)}} \text { with } \ell_{p}=\frac{1}{f} \sqrt{\frac{E(1-v)}{\rho(1-2 v)(1+v)}} \quad \ell_{s}=\frac{1}{f} \sqrt{\frac{E}{2 \rho(1+v)}}
$$

where $f$ is the wave frequency, $\ell_{p}$ and $\ell_{s}$ are the longitudinal and the shear wavelengths, respectively. Obviously, Eq. (22) shows that the longitudinal and the shear waves are coupled through the parameter $v$, i.e., Poisson's ratio. In other words, it is complicated to balance the stiffness and mass matrices using the same flexible integration points for both the longitudinal and shear waves. Due to this reason, we use lumped mass matrix (corresponding $\alpha=1$ in Eq. (18), as shown in Fig. 2c) to solve the propagation problem of the in-plane elastic wave in the solid [40]. It is noted that the lumped mass matrix is also a softened model compared with the consistent mass matrix [14]. Our previous work [34] has shown that with the mass-redistributed operation in the fluid domain together with the lumped mass matrix in the solid domain, the dispersion error can be minimized successfully compared with traditional Gauss integration points.

\subsection{Boundary conditions}

In this subsection, the Bloch boundary conditions are applied to Eq. (17) to solve the eigenvalues problem of the PCs within one periodic unit cell. These conditions 
with different arrangements including square and hexangular lattices are given based on the Floquet-Bloch theorem (as plotted in Fig. 1).

For the square lattice, the periodic boundary conditions of the fluid/solid PCs considering FSI are applied only on the elastic wave field, i.e. the displacements of the boundaries $\left(\Gamma_{1}, \Gamma_{3}\right)$ and $\left(\Gamma_{2}, \Gamma_{4}\right)$ of the unit cell should satisfy the form [32]:

$$
\begin{aligned}
& u_{x}\left(\mathbf{r}_{\Gamma_{1}}\right)=u_{x}\left(\mathbf{r}_{\Gamma_{3}}\right) e^{i k_{y} a}, u_{y}\left(\mathbf{r}_{\Gamma_{1}}\right)=u_{y}\left(\mathbf{r}_{\Gamma_{3}}\right) e^{i k_{y} a} \\
& u_{x}\left(\mathbf{r}_{\Gamma_{2}}\right)=u_{x}\left(\mathbf{r}_{\Gamma_{4}}\right) e^{i k_{x} a}, u_{y}\left(\mathbf{r}_{\Gamma_{2}}\right)=u_{y}\left(\mathbf{r}_{\Gamma_{4}}\right) e^{i k_{x} a}
\end{aligned}
$$

where $a$ is the lattice constant, the subscript in vector $\mathbf{r}$ denotes the detailed position, and $i^{2}=-1$.

For hexangular lattice, the boundary conditions of the fluid/solid PCs with FSI, i.e., $\left(\Gamma_{1}, \Gamma_{4}\right),\left(\Gamma_{2}, \Gamma_{5}\right)$ and $\left(\Gamma_{3}, \Gamma_{6}\right)$, are given as follows:

$$
\begin{aligned}
& u_{x}\left(\mathbf{r}_{\Gamma_{1}}\right)=u_{x}\left(\mathbf{r}_{\Gamma_{4}}\right) e^{-i\left(k_{x} \frac{a}{2}-k_{y} \frac{\sqrt{3} a}{2}\right)}, u_{y}\left(\mathbf{r}_{\Gamma_{1}}\right)=u_{y}\left(\mathbf{r}_{\Gamma_{4}}\right) e^{-i\left(k_{x} \frac{a}{2}-k_{y} \frac{\sqrt{3} a}{2}\right)} \\
& u_{x}\left(\mathbf{r}_{\Gamma_{2}}\right)=u_{x}\left(\mathbf{r}_{\Gamma_{5}}\right) e^{i\left(k_{x} \frac{a}{2}+k_{y} \frac{\sqrt{3} a}{2}\right)}, u_{y}\left(\mathbf{r}_{\Gamma_{2}}\right)=u_{y}\left(\mathbf{r}_{\Gamma_{5}}\right) e^{i\left(k_{x} \frac{a}{2}+k_{y} \frac{\sqrt{3} a}{2}\right)} \\
& u_{x}\left(\mathbf{r}_{\Gamma_{3}}\right)=u_{x}\left(\mathbf{r}_{\Gamma_{6}}\right) e^{i k_{x} a}, u_{y}\left(\mathbf{r}_{\Gamma_{3}}\right)=u_{y}\left(\mathbf{r}_{\Gamma_{6}}\right) e^{i k_{x} a}
\end{aligned}
$$

On the other hand, for the square lattice, the periodic boundary conditions for the solid/fluid PCs considering FSI are applied only on the fluid field, i.e. the pressures of the boundaries $\left(\Gamma_{1}, \Gamma_{3}\right)$ and $\left(\Gamma_{2}, \Gamma_{4}\right)$, of the unit cell are expressed by:

$$
p\left(\mathbf{r}_{\Gamma_{1}}\right)=p\left(\mathbf{r}_{\Gamma_{3}}\right) e^{i k_{y} a}, p\left(\mathbf{r}_{\Gamma_{2}}\right)=p\left(\mathbf{r}_{\Gamma_{4}}\right) e^{i k_{x} a}
$$

The periodic boundary conditions with respect to the boundaries $\left(\Gamma_{1}, \Gamma_{4}\right)$, $\left(\Gamma_{2}, \Gamma_{5}\right)$ and, $\left(\Gamma_{3}, \Gamma_{6}\right)$ of the unit cell with hexangular lattice are described by

$$
p\left(\mathbf{r}_{\Gamma_{1}}\right)=p\left(\mathbf{r}_{\Gamma_{4}}\right) e^{-i\left(k_{x} \frac{a}{2}-k_{y} \frac{\sqrt{3} a}{2}\right)}, p\left(\mathbf{r}_{\Gamma_{2}}\right)=p\left(\mathbf{r}_{\Gamma_{5}}\right) e^{i\left(k_{x} \frac{a}{2}+k_{y} \frac{\sqrt{3} a}{2}\right)}, p\left(\mathbf{r}_{\Gamma_{3}}\right)=p\left(\mathbf{r}_{\Gamma_{6}}\right) e^{i k_{x} a}
$$




\section{HUMR-FEM for fluid/solid and solid/fluid PCs with hybrid uncertainties}

The physic properties of a fluid/solid or solid/fluid PCs system considering FSI are usually characterized by its band structures and the pressure/displacement of mode shape. However, the uncertainties in the geometry, material, physics, manufacturing, measurement and installation are widespread in engineering, which may create a huge discrepancy between the design and manufacture. In such case, the physical behaviors are strongly affected by these uncertain factors. Thus, quantifying these influences enables us to understand the wave propagations as well as to conduct the design of acoustic/elastic wave devices $[41,42]$. In this section, the hybrid uncertainty analysis is herein addressed to solve the abovementioned problems.

\subsection{Foundation of hybrid uncertainty theory}

In the work [43], it is found that the material parameters are very important to control the bandgap of the solid/fluid PCs, indicating that both the solid and fluid phase materials parameters are very important. It is noted that the band structures of the PCs involving FSI are determined by solving the dispersion relations between the wave vector and the frequency. This is different from the natural frequency determinations in the traditional FSI system as the Bloch boundary conditions are required at the boundaries of the irreducible Brillion zone. In this case, the computational cost of PCs is very huge if the pure random is used to describe the uncertainty emerged in the PCs. Using the pure interval model may lead to an ultra-conservative result and is not economical. Since the PCs with FSI actually 
involve both types of uncertainties in the design. The hybrid uncertainty integrating the advantages of both the non-probabilistic and the probabilistic model may be more preferable. In this subsection, the interval random uncertainty is mathematically given.

In the interval random analysis, the uncertain parameter is defined though a series of variables $\mathbf{a}^{R}\left(\mathbf{b}^{I}\right)=\left[a_{1}^{R}\left(\mathbf{b}^{I}\right), a_{2}^{R}\left(\mathbf{b}^{I}\right), \ldots, a_{i}^{R}\left(\mathbf{b}^{I}\right), \ldots, a_{C}^{R}\left(\mathbf{b}^{I}\right)\right]$ (where $i=1,2,3, \ldots, C$ is the number of random variables) represents all the random variables $\mathbf{a}^{R}=\left[a_{1}^{R}, a_{2}^{R}, \ldots, a_{C}^{R}\right]$ and the interval parameter vector in the random type uncertainty is $\mathbf{b}^{I}=\left[\mathrm{b}_{1}^{I}, \mathrm{~b}_{2}^{I}, \ldots, \mathrm{b}_{C_{1}}^{I}\right]$ (where $C_{1}$ is the number of interval variables in each random variable). Thus, the expectation and the standard variance of interval random vector $\mathbf{a}^{R}\left(\mathbf{b}^{I}\right)$ can be written as [18]:

$$
\begin{aligned}
\operatorname{Exp}\left(\mathbf{a}^{R}\left(\mathbf{b}^{I}\right)\right) & =\operatorname{Exp}\left(a_{1}^{R}\left(\mathbf{b}^{I}\right), a_{2}^{R}\left(\mathbf{b}^{I}\right), \ldots, a_{C}^{R}\left(\mathbf{b}^{I}\right)\right) \\
& =\left[\left(\operatorname{Exp}\left(a_{1}^{R}\left(\mathbf{b}^{I}\right)\right), \operatorname{Exp}\left(a_{2}^{R}\left(\mathbf{b}^{I}\right)\right), \ldots, \operatorname{Exp}\left(a_{C}^{R}\left(\mathbf{b}^{I}\right)\right)\right)\right] \\
\operatorname{ED}\left(\mathbf{a}^{R}\left(\mathbf{b}^{I}\right)\right) & =\operatorname{ED}\left(a_{1}^{R}\left(\mathbf{b}^{I}\right), a_{2}^{R}\left(\mathbf{b}^{I}\right), \ldots, a_{C}^{R}\left(\mathbf{b}^{I}\right)\right)
\end{aligned}
$$

where $\operatorname{Exp}(\cdot)$ and $\operatorname{ED}(\cdot)$ denote the expectation and the standard variance.

In each random variable, its interval properties are defined as $[14,18]$ :

$$
\begin{gathered}
\mathbf{b}^{I}=[\underline{\mathbf{b}}, \overline{\mathbf{b}}]=\mathbf{b}^{m}+\Delta \mathbf{b}^{I} \delta, \Delta \mathbf{b}=(\overline{\mathbf{b}}-\underline{\mathbf{b}}) / 2, \delta \in[-1,1] \\
b_{l}^{I}=\left[\underline{b}_{l}, \bar{b}_{l}\right]=b_{l}^{m}+\Delta b_{l}^{I} \delta, \Delta b_{l}=\left(\bar{b}_{l}-\underline{b}_{l}\right) / 2, l \leq C_{1}
\end{gathered}
$$

where $\underline{\mathbf{b}}$ and $\overline{\mathbf{b}}$ denote the lower and upper bounds of $\mathbf{b}^{I} ; \mathbf{b}^{m}$ and $\Delta \mathbf{b}^{I}$ are the mean value vector and the interval uncertainty, respectively; $\underline{b}_{l}$ and $\bar{b}_{l}$ represent the lower and upper bounds of the $l$-th interval parameter $b_{l}^{I} ; b_{l}^{m}$ and $\Delta b_{l}$ are the mean value and the maximum deviation width of the interval parameter $b_{l}^{I}, \delta$ is the uncertain level. 
It should be noted that although the hybrid uncertainty of the PCs system with FSI has the new applied occasion in our work, the variables used to describe the interval random uncertainty are similar to those in the traditional FSI systems. To be more clearly, the interval random variables $a_{i}^{R}\left(\mathbf{b}^{I}\right)$ are modeled by the probability distribution function, whereas some of their distribution parameters are only given with limited information. For instance, if the Young's modulus is considered as an interval random variable that obeys the normal distribution with a bounded expectation, its probabilistic density function is shown in Fig. 3. (The expectation interval of the Young's modulus ranges from 67.45 to $74.55 \mathrm{GPa}$ with the standard variance $3.55 \mathrm{GPa})$.

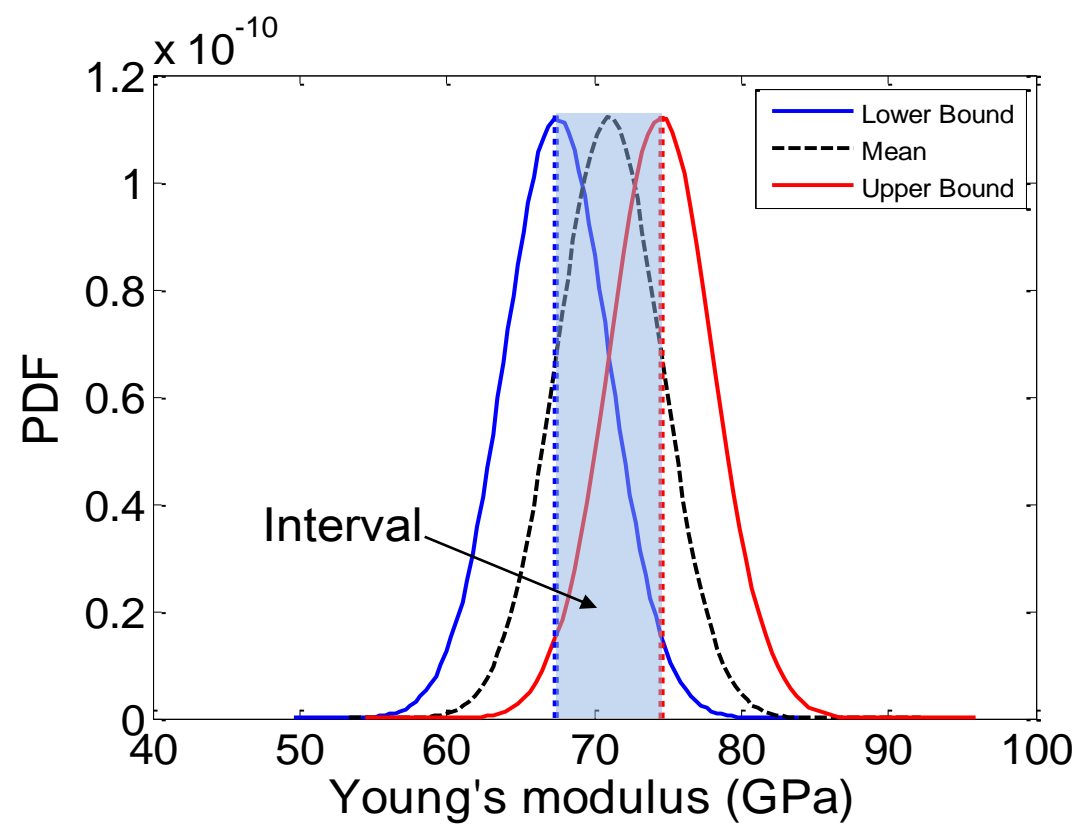

Fig. 3 The probabilistic density function (PDF) of the interval random model where the expectations of the Young's modulus are located in an assumed interval.

\subsection{Band structures problems with hybrid uncertain parameters}

In this subsection, we will present the HUMR-FEM approach to quantitively describe the hybrid uncertainty effect on the band structures. The eigenvalue problem 
of Eq. (17) involving uncertainty is given as [15,44]:

$$
\mathbf{K}\left(\mathbf{a}^{R}\left(\mathbf{b}^{I}\right)\right) \mathbf{U}_{j}=\varsigma_{j} \mathbf{M}^{\mathrm{MR}}\left(\mathbf{a}^{R}\left(\mathbf{b}^{I}\right)\right) \mathbf{U}_{j}, j=1,2, \ldots, m
$$

where $\varsigma_{j}=\omega_{j}^{2}$ is the $j$-th eigenvalue, $\mathbf{U}_{j}$ is the $j$-th eigenvector, $\mathbf{K}=\mathbf{K}\left(\mathbf{a}^{R}\left(\mathbf{b}^{I}\right)\right)$ and $\mathbf{M}^{\mathrm{MR}}=\mathbf{M}^{\mathrm{MR}}\left(\mathbf{a}^{R}\left(\mathbf{b}^{I}\right)\right)$ are the stiffness and the mass-redistributed matrices.

Using the Taylor series, the interval random eigenvalue $\varsigma_{j}=\varsigma_{j}\left(\mathbf{a}^{R}\left(\mathbf{b}^{I}\right)\right)$ can be firstly approximated at the expectation of the interval random parameters when the interval variable $\mathbf{b}^{I}$ is regarded as constant $\mathbf{b}$.

$$
\begin{aligned}
\varsigma_{j}\left(\mathbf{a}^{R}\left(\mathbf{b}^{I}\right)\right) & =\varsigma_{j}\left(\operatorname{Exp}\left(\mathbf{a}^{R}(\mathbf{b})\right)\right) \\
& +\sum_{i}^{C} \frac{\partial \varsigma_{j}\left(\operatorname{Exp}\left(a_{i}^{R}(\mathbf{b})\right)\right)}{\partial \operatorname{Exp}\left(a_{i}^{R}(\mathbf{b})\right)}\left(a_{i}^{R}(\mathbf{b})-\operatorname{Exp}\left(a_{i}^{R}(\mathbf{b})\right)\right) \\
& +\frac{1}{2 !} \sum_{i}^{C} \sum_{j}^{C} \frac{\partial^{2} \varsigma_{j}\left(\operatorname{Exp}\left(a_{i}^{R}(\mathbf{b}), a_{j}^{R}(\mathbf{b})\right)\right)}{\partial \operatorname{Exp}\left(a_{i}^{R}(\mathbf{b})\right) \partial E\left(a_{j}^{R}(\mathbf{b})\right)} \\
& \times\left(a_{i}^{R}(\mathbf{b})-\operatorname{Exp}\left(a_{i}^{R}(\mathbf{b})\right)\right)\left(a_{j}^{R}(\mathbf{b})-\operatorname{Exp}\left(a_{j}^{R}(\mathbf{b})\right)\right)+\ldots
\end{aligned}
$$

For the fluid/solid and solid/fluid PCs considering FSI, the improvement in accuracy obtained by using the higher order perturbation terms is rather small by taking account of the increase of the computational complexity and burden [21]. Neglecting the higher order perturbation terms yields the following simplified equation:

$$
\varsigma_{j}\left(\mathbf{a}^{R}\left(\mathbf{b}^{I}\right)\right)=\varsigma_{j}\left(\operatorname{Exp}\left(\mathbf{a}^{R}(\mathbf{b})\right)\right)+\sum_{i}^{c} \frac{\partial \varsigma_{j}\left(\operatorname{Exp}\left(a_{i}^{R}(\mathbf{b})\right)\right)}{\partial \operatorname{Exp}\left(a_{i}^{R}(\mathbf{b})\right)} \operatorname{ED}\left(a_{i}^{R}(\mathbf{b})\right)
$$

The random variables $\mathbf{a}^{R}$ related to the interval random vector $\mathbf{a}^{R}\left(\mathbf{b}^{I}\right)$ is secondly regarded as constant $\mathbf{a}$. Thus, both the expectation and standard variance are interval vectors, which can be further expanded by using the first-order Taylor series at the mean value $\mathbf{b}^{m}$ of the interval vector $\mathbf{b}^{I}$ based on Eq. (32). 


$$
\begin{aligned}
\varsigma_{j}\left(\mathbf{a}^{R}\left(\mathbf{b}^{I}\right)\right)= & \varsigma_{j}\left(\operatorname{Exp}\left(\mathbf{a}\left(\mathbf{b}^{m}\right)\right)\right)+\sum_{i}^{C} \sum_{l}^{C_{1}} \frac{\partial \varsigma_{j}\left(\operatorname{Exp}\left(\mathbf{a}\left(\mathbf{b}^{m}\right)\right)\right)}{\partial \operatorname{Exp}\left(a_{i}\left(\mathbf{b}^{m}\right)\right)} \frac{\partial \operatorname{Exp}\left(a_{i}\left(\mathbf{b}^{m}\right)\right)}{\partial b_{l}^{I}} \Delta b_{l} \delta \\
& +\sum_{i}^{c} \frac{\partial \varsigma_{j}\left(\operatorname{Exp}\left(\mathbf{a}\left(\mathbf{b}^{m}\right)\right)\right)}{\partial \operatorname{Exp}\left(a_{i}\left(\mathbf{b}^{m}\right)\right)} \cdot \operatorname{ED}\left(a_{i}\left(\mathbf{b}^{m}\right)\right) \\
& +\sum_{i}^{C} \sum_{l}^{c_{1}} \frac{\partial \varsigma_{j}\left(\operatorname{Exp}\left(\mathbf{a}\left(\mathbf{b}^{m}\right)\right)\right)}{\partial \operatorname{Exp}\left(a_{i}\left(\mathbf{b}^{m}\right)\right)} \frac{\partial \operatorname{ED}\left(a_{i}\left(\mathbf{b}^{m}\right)\right)}{\partial b_{l}^{I}} \Delta b_{l}^{I} \delta \\
& +\sum_{i}^{C} \sum_{l}^{C_{l}} \frac{\partial^{2} \varsigma_{j}\left(\operatorname{Exp}\left(\mathbf{a}\left(\mathbf{b}^{m}\right)\right)\right)}{\partial \operatorname{Exp}\left(a_{i}\left(\mathbf{b}^{m}\right)\right)^{2}} \frac{\partial \operatorname{Exp}\left(a_{i}\left(\mathbf{b}^{m}\right)\right)}{\partial b_{l}^{I}} \Delta b_{l}^{I} \delta \cdot \operatorname{ED}\left(a_{i}\left(\mathbf{b}^{m}\right)\right)
\end{aligned}
$$

where $\partial \varsigma_{j}\left(\operatorname{Exp}\left(\mathbf{a}\left(\mathbf{b}^{m}\right)\right)\right) / \partial \operatorname{Exp}\left(a_{i}\left(\mathbf{b}^{m}\right)\right)$ and $\partial^{2} \varsigma_{j}\left(\operatorname{Exp}\left(\mathbf{a}\left(\mathbf{b}^{m}\right)\right)\right) / \partial \operatorname{Exp}\left(a_{i}\left(\mathbf{b}^{m}\right)\right)^{2}$ denote the first and second order derivatives of the $j$-th eigenvalue with respect to the expectation of the interval random variables, respectively. The expectation and the standard variance are the explicit functions of the interval uncertain parameters, thus the corresponding derivatives can be solved based on the different random types.

To obtain the interval random vector of the $j$-th eigenvalue, the first and second order derivatives of the $j$-th eigenvalue with respect to the expectation of the interval random variables are still to be calculated. Taking the derivative of Eq. (30) with respect to the expectation of the interval random parameter yields

$$
\begin{aligned}
& {\left[\mathbf{K}-\varsigma_{j}\left(\operatorname{Exp}\left(a_{i}\left(\mathbf{b}^{m}\right)\right)\right) \mathbf{M}^{\mathrm{MR}}\right] \frac{\partial \mathbf{U}_{j}}{\partial \operatorname{Exp}\left(a_{i}\left(\mathbf{b}^{m}\right)\right)}-\mathbf{M}^{\mathrm{MR}} \mathbf{U}_{j} \frac{\partial \varsigma_{j}\left(\operatorname{Exp}\left(a_{i}\left(\mathbf{b}^{m}\right)\right)\right)}{\partial \operatorname{Exp}\left(a_{i}\left(\mathbf{b}^{m}\right)\right)}} \\
& =-\left[\frac{\partial \mathbf{K}}{\partial \operatorname{Exp}\left(a_{i}\left(\mathbf{b}^{m}\right)\right)}-\varsigma_{j} \frac{\partial \mathbf{M}^{\mathrm{MR}}}{\partial \operatorname{Exp}\left(a_{i}\left(\mathbf{b}^{m}\right)\right)}\right] \mathbf{U}_{j}
\end{aligned}
$$

By using the normalization condition $\mathbf{U}_{j}^{\mathrm{T}} \mathbf{M}^{\mathrm{MR}} \mathbf{U}_{j}=1$ to Eq. (34), the first derivative of $j$-th eigenvalue with respect to the expectation $a_{i}(\mathbf{b})$ at the expectation $\operatorname{Exp}\left(a_{i}(\mathbf{b})\right)$ can be calculated as: 


$$
\frac{\partial \varsigma_{j}\left(\operatorname{Exp}\left(\mathbf{a}\left(\mathbf{b}^{m}\right)\right)\right)}{\partial \operatorname{Exp}\left(a_{i}\left(\mathbf{b}^{m}\right)\right)}=\mathbf{U}_{j}^{\mathrm{T}}\left[\frac{\partial \mathbf{K}}{\partial \operatorname{Exp}\left(a_{i}\left(\mathbf{b}^{m}\right)\right)}-\varsigma_{j}\left(\operatorname{Exp}\left(\mathbf{a}\left(\mathbf{b}^{m}\right)\right)\right) \frac{\partial \mathbf{M}^{\mathrm{MR}}}{\partial \operatorname{Exp}\left(a_{i}\left(\mathbf{b}^{m}\right)\right)}\right] \mathbf{U}_{j}
$$

Taking the derivative of Eq. (34) with respect to the interval vector $\mathbf{b}$ at the mean value $\mathbf{b}^{m}$, and pre-multiplying $\mathbf{U}_{j}^{\mathrm{T}}$ yields:

$$
\begin{aligned}
\frac{\partial^{2} \varsigma_{j}\left(\operatorname{Exp}\left(\mathbf{a}\left(\mathbf{b}^{m}\right)\right)\right)}{\partial \operatorname{Exp}\left(a_{i}\left(\mathbf{b}^{m}\right)\right)^{2}} & =\mathbf{U}_{j}^{\mathrm{T}}\left[\frac{\partial \mathbf{K}}{\partial \operatorname{Exp}\left(a_{i}\left(\mathbf{b}^{m}\right)\right)}-\varsigma_{j} \frac{\partial \mathbf{M}^{\mathrm{MR}}}{\partial \operatorname{Exp}\left(a_{i}\left(\mathbf{b}^{m}\right)\right)}\right] \frac{\partial \mathbf{U}_{j}}{\partial \operatorname{Exp}\left(a_{i}\left(\mathbf{b}^{m}\right)\right)} \\
& -\mathbf{U}_{j}^{\mathrm{T}}\left[\frac{\partial \varsigma_{j}\left(\operatorname{Exp}\left(\mathbf{a}\left(\mathbf{b}^{m}\right)\right)\right)}{\partial \operatorname{Exp}\left(a_{i}\left(\mathbf{b}^{m}\right)\right)} \mathbf{M}^{\mathrm{MR}}\right] \frac{\partial \mathbf{U}_{j}}{\partial \operatorname{Exp}\left(a_{i}\left(\mathbf{b}^{m}\right)\right)} \\
& -\mathbf{U}_{j}^{\mathrm{T}}\left[\frac{\partial \varsigma_{j}\left(\operatorname{Exp}\left(\mathbf{a}\left(\mathbf{b}^{m}\right)\right)\right)}{\partial \operatorname{Exp}\left(a_{i}\left(\mathbf{b}^{m}\right)\right)} \frac{\partial \mathbf{M}^{\mathrm{MR}}}{\partial \operatorname{Exp}\left(a_{i}\left(\mathbf{b}^{m}\right)\right)}\right] \mathbf{U}_{j}
\end{aligned}
$$

where the derivative $\partial \mathbf{U}_{j} / \partial \operatorname{Exp}\left(a_{i}\left(\mathbf{b}^{m}\right)\right)$ denotes the $j$-th eigenvector $\mathbf{U}_{j}$ with respect to the expectation of $a_{i}(\mathbf{b})$, the derivative computation is introduced in the following subsection. For the band structures problems, it should be emphasized that Eq. (36) is capable of dealing with the problem with distinct eigenvalues and distinct derivatives; the computation of the repeated case of eigenvalue and its derivatives can be seen in Ref. [44]. Once Eqs. (35) and (36) are computed, Eq. (33) can be solved to determine the hybrid uncertainty effect on the band diagrams.

\subsection{Statistical characteristics of the extreme bounds of the bandgaps}

In the above subsection, the band structures are interval random variables due to the twice expansion, therefore, the band structures problem involving hybrid uncertainty is transformed into a linear function of the random variables and the interval variables. The statistical characteristics including the expectation and the standard variance of the eigenvalue denote the random property, the extreme bounds including the lower and upper bounds represent the interval property. In this subsection, 
the expectation and the standard variance of the extreme bounds are computed.

\subsubsection{Extreme bounds of the band structures}

Taking the first order derivative of the eigenvalue with respect to the increment $\Delta b_{l}$, the sensitivity of the interval variable can be determined as:

$$
\begin{aligned}
\frac{\partial \varsigma_{j}\left(\mathbf{a}^{R}\left(\mathbf{b}^{I}\right)\right)}{\partial \Delta b_{l}^{I}} & =\sum_{i}^{C} \sum_{l}^{C_{1}} \frac{\partial \varsigma_{j}\left(\operatorname{Exp}\left(\mathbf{a}\left(\mathbf{b}^{m}\right)\right)\right)}{\partial \operatorname{Exp}\left(a_{i}\left(\mathbf{b}^{m}\right)\right)} \frac{\partial \operatorname{Exp}\left(a_{i}\left(\mathbf{b}^{m}\right)\right)}{\partial b_{l}^{I}} \delta \\
& +\sum_{i}^{C} \sum_{l}^{C_{1}} \frac{\partial \varsigma_{j}\left(\operatorname{Exp}\left(\mathbf{a}\left(\mathbf{b}^{m}\right)\right)\right)}{\partial \operatorname{Exp}\left(a_{i}\left(\mathbf{b}^{m}\right)\right)} \frac{\partial \operatorname{ED}\left(a_{i}\left(\mathbf{b}^{m}\right)\right)}{\partial b_{l}^{I}} \delta \\
& +\sum_{i}^{C} \sum_{l}^{C_{1}} \frac{\partial^{2} \varsigma_{j}\left(\operatorname{Exp}\left(\mathbf{a}\left(\mathbf{b}^{m}\right)\right)\right)}{\partial \operatorname{Exp}\left(a_{i}\left(\mathbf{b}^{m}\right)\right)^{2}} \frac{\partial \operatorname{Exp}\left(a_{i}\left(\mathbf{b}^{m}\right)\right)}{\partial b_{l}^{I}} \cdot \operatorname{ED}\left(a_{i}\left(\mathbf{b}^{m}\right)\right) \delta
\end{aligned}
$$

Obviously, the sensitivity of the eigenvalue with respect to the interval increment is a constant and independent of the interval variables, so the eigenvalue is a monotonic function of $\Delta b_{l}$. Using the vertex method [21], the vertex of $\Delta b_{l}$ can be written as $\Delta \hat{b}_{l}= \pm\left|\Delta b_{l}\right|$. Because the number of the interval variables is $l_{1}+l_{2}+\ldots+l_{\text {in }}+\ldots$ (where $l_{\text {in }}$ denotes the number of interval variable in the $i$-th random parameter), thus, there are $L=l_{1} \cdot l_{2} \cdots l_{i n}$ combinations of interval variables based. Each combination can generate an eigenvalue $\zeta_{j, N}($ where $N=1,2, \ldots, L)$ :

$$
\begin{aligned}
\varsigma_{j, N}\left(\mathbf{a}^{R}\left(\mathbf{b}^{I}\right)\right) & =\varsigma_{j}\left(\operatorname{Exp}\left(\mathbf{a}\left(\mathbf{b}^{m}\right)\right)\right)+\sum_{i}^{c} \sum_{l}^{c_{1}} \frac{\partial \varsigma_{j}\left(\operatorname{Exp}\left(\mathbf{a}\left(\mathbf{b}^{m}\right)\right)\right)}{\partial \operatorname{Exp}\left(a_{i}\left(\mathbf{b}^{m}\right)\right)} \frac{\partial \operatorname{Exp}\left(a_{i}\left(\mathbf{b}^{m}\right)\right)}{\partial b_{l}^{I}} \Delta \hat{b}_{l, N}^{I} \delta \\
& +\sum_{i}^{C} \frac{\partial \varsigma_{j}\left(\operatorname{Exp}\left(\mathbf{a}\left(\mathbf{b}^{m}\right)\right)\right)}{\partial \operatorname{Exp}\left(a_{i}\left(\mathbf{b}^{m}\right)\right)} \cdot \operatorname{ED}\left(a_{i}\left(\mathbf{b}^{m}\right)\right) \\
& +\sum_{i}^{C} \sum_{l}^{c_{1}} \frac{\partial \varsigma_{j}\left(\operatorname{Exp}\left(\mathbf{a}\left(\mathbf{b}^{m}\right)\right)\right)}{\partial \operatorname{Exp}\left(a_{i}\left(\mathbf{b}^{m}\right)\right)} \frac{\partial \operatorname{ED}\left(a_{i}\left(\mathbf{b}^{m}\right)\right)}{\partial b_{l}^{I}} \Delta \hat{b}_{l, N}^{I} \delta \\
& +\sum_{i}^{C} \sum_{l}^{C_{1}} \frac{\partial^{2} \varsigma_{j}\left(\operatorname{Exp}\left(\mathbf{a}\left(\mathbf{b}^{m}\right)\right)\right)}{\partial \operatorname{Exp}\left(a_{i}\left(\mathbf{b}^{m}\right)\right)^{2}} \frac{\partial \operatorname{Exp}\left(a_{i}\left(\mathbf{b}^{m}\right)\right)}{\partial b_{l}^{I}} \Delta \hat{b}_{l, N}^{I} \delta \cdot \operatorname{ED}\left(a_{i}\left(\mathbf{b}^{m}\right)\right)
\end{aligned}
$$


Consequently, the lower and upper bounds of the eigenvalue can be expressed as:

$$
\underline{\varsigma}_{j}=\min _{\substack{N=1,2, \ldots, L \\ L=l_{1} l_{2}, \ldots, l_{i n}}}\left\{\varsigma_{j, N}\left(\mathbf{a}^{R}\left(\mathbf{b}^{I}\right)\right)\right\}, \bar{\varsigma}_{j}=\max _{\substack{N=1,2, \ldots, L \\ L=l_{1} l_{2}, \ldots, l_{i n}}}\left\{\varsigma_{j, N}\left(\mathbf{a}^{R}\left(\mathbf{b}^{I}\right)\right)\right\}
$$

It should be emphasized that the interval variable is considered as the vertex value in each combination. Thus, the interval random variables are transformed into the random variables whose expectation and standard variance are interval variables.

3.3.2 Extreme bounds of the expectations and standard variances of the band structures

As mentioned above, the extreme bounds in Eq. (39) are the function of random variables, thus the expectations can be derived through:

$$
\begin{aligned}
& \operatorname{Exp}\left(\underline{\varsigma}_{j}\right)=\min _{\substack{N=1,2, \ldots, L \\
L=l_{l} l_{2}, \ldots, l_{\text {in }}}}\left\{\varsigma_{j}\left(\operatorname{Exp}\left(\mathbf{a}\left(\mathbf{b}^{m}\right)\right)\right)+\sum_{i}^{c} \sum_{l}^{C_{1}} \frac{\partial \varsigma_{j}\left(\operatorname{Exp}\left(\mathbf{a}\left(\mathbf{b}^{m}\right)\right)\right)}{\partial \operatorname{Exp}\left(a_{i}\left(\mathbf{b}^{m}\right)\right)} \frac{\partial \operatorname{Exp}\left(a_{i}\left(\mathbf{b}^{m}\right)\right)}{\partial b_{l}^{I}} \Delta \hat{b}_{l, N}^{I} \delta\right\} \\
& \operatorname{Exp}\left(\bar{\varsigma}_{j}\right)=\max _{\substack{N=1,2, \ldots, L \\
L=l_{l} l_{2}, \ldots, l_{i n}}}\left\{\varsigma_{j}\left(\operatorname{Exp}\left(\mathbf{a}\left(\mathbf{b}^{m}\right)\right)\right)+\sum_{i}^{C} \sum_{l}^{C_{1}} \frac{\partial \varsigma_{j}\left(\operatorname{Exp}\left(\mathbf{a}\left(\mathbf{b}^{m}\right)\right)\right)}{\partial \operatorname{Exp}\left(a_{i}\left(\mathbf{b}^{m}\right)\right)} \frac{\partial \operatorname{Exp}\left(a_{i}\left(\mathbf{b}^{m}\right)\right)}{\partial b_{l}^{I}} \Delta \hat{b}_{l, N}^{I} \delta\right\}
\end{aligned}
$$

where $\operatorname{Exp}\left(\underline{\varsigma}_{j}\right)$ and $\operatorname{Exp}\left(\bar{\varsigma}_{j}\right)$ represent the expectation of the lower and upper bounds of the eigenvalue, respectively. As the expectations of the extreme bounds denote the interval distributions of the expectations, in other words, these expectations can also be considered as the extreme bounds of the expectations. Thus, the interval distribution of the expectation can be expressed as:

$$
\operatorname{Exp}^{I}\left(\varsigma_{j}\right)=\left[\operatorname{Exp}\left(\underline{\varsigma}_{j}\right), \operatorname{Exp}\left(\bar{\varsigma}_{j}\right)\right]
$$

Similarly, the standard variance can be derived through: 


$$
\begin{aligned}
& \operatorname{ED}\left(\underline{\varsigma}_{j}\right)=\min _{\substack{N=1,2, \ldots \\
L=l_{1}, l_{2}, \cdots \\
l_{i n}}}\left\{\begin{array}{l}
\sum_{i}^{c} \frac{\partial \varsigma_{j}\left(\operatorname{Exp}\left(\mathbf{a}\left(\mathbf{b}^{m}\right)\right)\right)}{\partial \operatorname{Exp}\left(a_{i}\left(\mathbf{b}^{m}\right)\right)} \cdot \operatorname{ED}\left(a_{i}\left(\mathbf{b}^{m}\right)\right) \\
+\sum_{l}^{C} \frac{\partial \varsigma_{j}\left(\operatorname{Exp}\left(\mathbf{a}\left(\mathbf{b}^{m}\right)\right)\right)}{\partial \operatorname{Exp}\left(a_{i}\left(\mathbf{b}^{m}\right)\right)} \frac{\partial \operatorname{ED}\left(a_{i}\left(\mathbf{b}^{m}\right)\right)}{\partial b_{l}^{I}} \Delta \hat{b}_{l, N}^{I} \delta \\
+\sum_{i}^{C} \sum_{l}^{C_{1}} \frac{\partial^{2} \varsigma_{j}\left(\operatorname{Exp}\left(\mathbf{a}\left(\mathbf{b}^{m}\right)\right)\right)}{\partial \operatorname{Exp}\left(a_{i}\left(\mathbf{b}^{m}\right)\right)^{2}} \frac{\partial \operatorname{Exp}\left(a_{i}\left(\mathbf{b}^{m}\right)\right)}{\partial b_{l}^{I}} \Delta \hat{b}_{l, N}^{I} \delta \cdot \operatorname{ED}\left(a_{i}\left(\mathbf{b}^{m}\right)\right)
\end{array}\right\} \\
& \operatorname{ED}\left(\bar{\varsigma}_{j}\right)=\max _{\substack{N=1,2, \ldots, L \\
L=l_{1}, l_{2}, l_{i n}}}\left\{\begin{array}{l}
\sum_{i}^{c} \frac{\partial \varsigma_{j}\left(\operatorname{Exp}\left(\mathbf{a}\left(\mathbf{b}^{m}\right)\right)\right)}{\partial \operatorname{Exp}\left(a_{i}\left(\mathbf{b}^{m}\right)\right)} \cdot \operatorname{ED}\left(a_{i}\left(\mathbf{b}^{m}\right)\right) \\
\sum_{l}^{c} \frac{\partial \varsigma_{j}\left(\operatorname{Exp}\left(\mathbf{a}\left(\mathbf{b}^{m}\right)\right)\right)}{\partial \operatorname{Exp}\left(a_{i}\left(\mathbf{b}^{m}\right)\right)} \frac{\partial \operatorname{ED}\left(a_{i}\left(\mathbf{b}^{m}\right)\right)}{\partial b_{l}^{I}} \Delta \hat{b}_{l, N}^{I} \delta \\
+\sum_{i}^{c} \sum_{l}^{C_{1}} \frac{\partial^{2} \varsigma_{j}\left(\operatorname{Exp}\left(\mathbf{a}\left(\mathbf{b}^{m}\right)\right)\right)}{\partial \operatorname{Exp}\left(a_{i}\left(\mathbf{b}^{m}\right)\right)^{2}} \frac{\partial \operatorname{Exp}\left(a_{i}\left(\mathbf{b}^{m}\right)\right)}{\partial b_{l}^{I}} \Delta \hat{b}_{l, N}^{I} \delta \cdot \operatorname{ED}\left(a_{i}\left(\mathbf{b}^{m}\right)\right)
\end{array}\right\}
\end{aligned}
$$

where $E D\left(\underline{\varsigma}_{j}\right)$ and $E D\left(\bar{\varsigma}_{j}\right)$ represent the standard variance of the lower and upper bounds of the eigenvalue. Following Eq. (41), the lower and upper bounds of the standard variance can be written as:

$$
E D^{I}\left(\varsigma_{j}\right)=\left[E D\left(\underline{\varsigma}_{j}\right), E D\left(\bar{\varsigma}_{j}\right)\right]
$$

Based on Eqs. (39), (41) and (43), the hybrid model with the interval random properties for the eigenvalue problem are depicted.

\subsection{Mode shape problem with hybrid uncertain parameters}

In this subsection, the influences of the hybrid uncertainty on the mode shape which represents the manifestation of the eigenvector is studied. Similar to Eq. (33), the expansion of the eigenvector at the expectation of the interval random parameter and the mean value of the interval parameter can be expressed as 


$$
\begin{aligned}
\mathbf{U}_{j}\left(\mathbf{a}^{R}\left(\mathbf{b}^{I}\right)\right) & =\mathbf{U}_{j}\left(\operatorname{Exp}\left(\mathbf{a}\left(\mathbf{b}^{m}\right)\right)\right)+\sum_{i}^{c} \sum_{l}^{C_{1}} \frac{\partial \mathbf{U}_{j}\left(\operatorname{Exp}\left(\mathbf{a}\left(\mathbf{b}^{m}\right)\right)\right)}{\partial \operatorname{Exp}\left(a_{i}\left(\mathbf{b}^{m}\right)\right)} \frac{\partial \operatorname{Exp}\left(a_{i}\left(\mathbf{b}^{m}\right)\right)}{\partial b_{l}^{I}} \Delta b_{l} \delta \\
& +\sum_{i}^{c} \frac{\partial \mathbf{U}_{j}\left(\operatorname{Exp}\left(\mathbf{a}\left(\mathbf{b}^{m}\right)\right)\right)}{\partial \operatorname{Exp}\left(a_{i}\left(\mathbf{b}^{m}\right)\right)} \cdot \operatorname{ED}\left(a_{i}\left(\mathbf{b}^{m}\right)\right) \\
& +\sum_{i}^{C} \sum_{l}^{C_{1}} \frac{\partial \mathbf{U}_{j}\left(\operatorname{Exp}\left(\mathbf{a}\left(\mathbf{b}^{m}\right)\right)\right)}{\partial \operatorname{Exp}\left(a_{i}\left(\mathbf{b}^{m}\right)\right)} \frac{\partial \operatorname{ED}\left(a_{i}\left(\mathbf{b}^{m}\right)\right)}{\partial b_{l}^{I}} \Delta b_{l}^{I} \delta \\
& +\sum_{i}^{C} \sum_{l}^{C_{1}} \frac{\partial^{2} \mathbf{U}_{j}\left(\operatorname{Exp}\left(\mathbf{a}\left(\mathbf{b}^{m}\right)\right)\right)}{\partial \operatorname{Exp}\left(a_{i}\left(\mathbf{b}^{m}\right)\right)^{2}} \frac{\partial \operatorname{Exp}\left(a_{i}\left(\mathbf{b}^{m}\right)\right)}{\partial b_{l}^{I}} \Delta b_{l}^{I} \delta \cdot \operatorname{ED}\left(a_{i}\left(\mathbf{b}^{m}\right)\right)
\end{aligned}
$$

The derivative of the $j$-th eigenvector with respect to the expectation of the interval uncertain parameter is computed as [44]:

$$
\frac{\partial \mathbf{U}_{j}\left(\operatorname{Exp}\left(\mathbf{a}\left(\mathbf{b}^{m}\right)\right)\right)}{\partial \operatorname{Exp}\left(a_{i}\left(\mathbf{b}^{m}\right)\right)}=\mathbf{V}_{j}+\mathbf{U}_{j} \theta_{j}, \quad \mathbf{U}_{j}^{\mathrm{T}} \mathbf{M}^{\mathrm{MR}} \theta_{j}=0
$$

where $\theta_{j}$ is a known constant coefficient for calculating the homogenous solutions, $\mathbf{V}_{j}$ is the particular solution, which satisfies the orthogonality with $\mathbf{U}_{j}^{\mathrm{T}}$. The following extended system can be formulated to solve the particular solution $\mathbf{V}_{j}$ :

$$
\left[\begin{array}{cc}
\mathbf{K}-\varsigma_{j} \mathbf{M}^{\mathrm{MR}} & \gamma \mathbf{M}^{\mathrm{MR}} \mathbf{U}_{j} \\
-\gamma \mathbf{U}_{j}^{\mathrm{T}} \mathbf{M}^{\mathrm{MR}} & 0
\end{array}\right]\left[\begin{array}{c}
\mathbf{V}_{j} \\
\gamma^{-1} \frac{\partial \varsigma_{j}}{\partial \operatorname{Exp}\left(a_{i}\left(\mathbf{b}^{m}\right)\right)}
\end{array}\right]=\left[\begin{array}{c}
\frac{\partial \mathbf{K}}{\partial \operatorname{Exp}\left(a_{i}\left(\mathbf{b}^{m}\right)\right)}-\frac{\varsigma_{j} \partial \mathbf{M}^{\mathrm{MR}}}{\partial \operatorname{Exp}\left(a_{i}\left(\mathbf{b}^{m}\right)\right)} \\
0
\end{array}\right]
$$

where $\gamma$ is determined through dividing the largest element of $\left(\mathbf{K}-\varsigma_{j} \mathbf{M}^{\mathrm{MR}}\right)$ by the largest element of $\mathbf{M}^{\mathrm{MR}} \mathbf{U}_{j}$.

The constant $\theta_{j}$ in Eq. (45) can be determined by differentiating the normalized constant Eq. (35):

$$
\theta_{j}=-0.5 \mathbf{U}_{j}^{\mathrm{T}} \frac{\partial \mathbf{M}^{\mathrm{MR}}\left(\operatorname{Exp}\left(\mathbf{a}\left(\mathbf{b}^{m}\right)\right)\right)}{\partial \operatorname{Exp}\left(a_{i}\left(\mathbf{b}^{m}\right)\right)} \mathbf{U}_{j}, \quad \mathbf{U}_{j}^{\mathrm{T}} \mathbf{M}^{\mathrm{MR}} \theta_{j}=0
$$

To compute the interval random eigenvector, the second derivative of the 
eigenvector with respect to the expectation of the hybrid uncertain parameter is still to be calculated. Here, an efficient solution is given to deal with it, which is partly based on Ref. [44]. Rearranging Eq. (30), we can obtain:

$$
\mathbf{\Phi} \mathbf{U}=\mathbf{U} \Lambda
$$

where $\boldsymbol{\Phi}=\mathbf{M}^{\mathrm{MR}}\left(\mathbf{a}^{R}\left(\mathbf{b}^{I}\right)\right)^{-1} \mathbf{K}\left(\mathbf{a}^{R}\left(\mathbf{b}^{I}\right)\right) \quad$ and $\quad \boldsymbol{\Lambda}=\left[\varsigma_{1}, \varsigma_{2}, \ldots, \varsigma_{m}\right] . \quad$ Taking once differential for Eq. (48) yields:

$$
\left(\boldsymbol{\Phi}-\varsigma_{j} \mathbf{I}\right) \frac{\partial \mathbf{U}_{j}}{\partial \operatorname{Exp}\left(a_{i}\left(\mathbf{b}^{m}\right)\right)}=\frac{\partial \varsigma_{j}}{\partial \operatorname{Exp}\left(a_{i}\left(\mathbf{b}^{m}\right)\right)} \mathbf{U}_{j}-\frac{\partial \boldsymbol{\Phi}}{\partial \operatorname{Exp}\left(a_{i}\left(\mathbf{b}^{m}\right)\right)} \mathbf{U}_{j}
$$

where $\mathbf{I}$ is the identity matrix.

Typically, the eigenvector solution is not unique for the eigen problem defined in Eq. (48), because when the eigenvector is multiplied or divided by any scaler, the eigensystem equation Eq. (48) is still satisfied. Here, it is need to introduce another normalization condition to ensure the uniqueness of the eigenvectors:

$$
\left\{\mathbf{U}\left(\mathbf{a}^{R}\left(\mathbf{b}^{I}\right)\right)_{j}\right\}_{n_{i}}=1
$$

where $\left\{\bullet_{j}\right\}_{n_{i}}$ is the $n_{i}$-th component of the $j$-th eigenvector, of which the selection makes such a case that the product of the absolute values of the corresponding components in the eigenvectors is the largest. Differentiating Eq. (50) yields:

$$
\left\{\frac{\partial \mathbf{U}_{j}}{\partial \operatorname{Exp}\left(a_{i}\left(\mathbf{b}^{m}\right)\right)}\right\}_{n_{i}}=0 \text { or } \mathbf{W}_{i} \frac{\partial \mathbf{U}_{j}}{\partial \operatorname{Exp}\left(a_{i}\left(\mathbf{b}^{m}\right)\right)}=0, i=1,2, \ldots, n_{i}
$$

where $\mathbf{W}_{i}$ is a weighting vector with dimension of $1 \times n$ and of which the $n_{i}$-th component is 1 while the rest components are set to 0 .

Taking once differential for Eq. (49) with respect to the expectation of the interval random parameter and taking once differential for Eq. (50), we can obtain the 
augmented equation as follows:

$$
\left[\begin{array}{cc}
\mathbf{\Phi}-\varsigma_{j} \mathbf{I} & -\mathbf{U}_{j} \\
\mathbf{W}_{j} & 0
\end{array}\right]\left\{\begin{array}{c}
\frac{\partial^{2} \mathbf{U}_{j}}{\partial \operatorname{Exp}\left(a_{i}\left(\mathbf{b}^{m}\right)\right)^{2}} \\
\frac{\partial^{2} \varsigma_{j}}{\partial \operatorname{Exp}\left(a_{i}\left(\mathbf{b}^{m}\right)\right)^{2}}
\end{array}\right\}=\left\{\begin{array}{c}
2\left(-\frac{\partial \mathbf{\Phi}}{\partial \operatorname{Exp}\left(a_{i}\left(\mathbf{b}^{m}\right)\right)}+\frac{\partial \varsigma_{j}}{\partial \operatorname{Exp}\left(a_{i}\left(\mathbf{b}^{m}\right)\right)} \mathbf{I}\right) \\
\frac{\partial \mathbf{U}_{j}}{\partial \operatorname{Exp}\left(a_{i}\left(\mathbf{b}^{m}\right)\right)}-\frac{\partial^{2} \mathbf{\Phi}}{\partial \operatorname{Exp}\left(a_{i}\left(\mathbf{b}^{m}\right)\right)^{2}} \mathbf{U}_{j} \\
0
\end{array}\right\}
$$

By computing Eq. (52), we can get the second derivative of the eigenvector with respect to the expectation of the hybrid uncertain parameter. Once Eqs. (45) and (52) are determined, Eq. (44) can be calculated.

\subsection{Statistical characteristics of the extreme bounds of the mode shape}

\subsubsection{Extreme bounds of the mode shape}

Taking the first order derivative of the eigenvector with respect to the increment $\Delta b_{l}$, the gradient of the interval variable can be determined as:

$$
\begin{aligned}
\frac{\partial \mathbf{U}_{j}\left(\mathbf{a}^{R}\left(\mathbf{b}^{I}\right)\right)}{\partial \Delta b_{l}^{I}} & =\sum_{i}^{c} \sum_{l}^{C_{1}} \frac{\partial \mathbf{U}_{j}\left(\operatorname{Exp}\left(\mathbf{a}\left(\mathbf{b}^{m}\right)\right)\right)}{\partial \operatorname{Exp}\left(a_{i}\left(\mathbf{b}^{m}\right)\right)} \frac{\partial \operatorname{Exp}\left(a_{i}\left(\mathbf{b}^{m}\right)\right)}{\partial b_{l}^{I}} \delta \\
& +\sum_{i}^{C} \sum_{l}^{C_{1}} \frac{\partial \mathbf{U}_{j}\left(\operatorname{Exp}\left(\mathbf{a}\left(\mathbf{b}^{m}\right)\right)\right)}{\partial \operatorname{Exp}\left(a_{i}\left(\mathbf{b}^{m}\right)\right)} \frac{\partial \operatorname{ED}\left(a_{i}\left(\mathbf{b}^{m}\right)\right)}{\partial b_{l}^{I}} \delta \\
& +\sum_{i}^{C} \sum_{l}^{C_{1}} \frac{\partial^{2} \mathbf{U}_{j}\left(\operatorname{Exp}\left(\mathbf{a}\left(\mathbf{b}^{m}\right)\right)\right)}{\partial \operatorname{Exp}\left(a_{i}\left(\mathbf{b}^{m}\right)\right)^{2}} \frac{\partial \operatorname{Exp}\left(a_{i}\left(\mathbf{b}^{m}\right)\right)}{\partial b_{l}^{I}} \delta \cdot \operatorname{ED}\left(a_{i}\left(\mathbf{b}^{m}\right)\right)
\end{aligned}
$$

Eq. (53) shows that the $j$-th eigenvalue changes monotonously versus $\Delta b_{l}$. Using the vertex relationship with $\Delta \hat{b}_{l}= \pm\left|\Delta b_{l}\right|$, each combination can generate an eigenvector $\mathbf{U}_{j, N}($ where $N=1,2, \ldots, L)$ as: 


$$
\begin{aligned}
& \mathbf{U}_{j, N}\left(\mathbf{a}^{R}\left(\mathbf{b}^{I}\right)\right)=\mathbf{U}_{j}\left(\operatorname{Exp}\left(\mathbf{a}\left(\mathbf{b}^{m}\right)\right)\right)+\sum_{i}^{C} \frac{\partial \mathbf{U}_{j}\left(\operatorname{Exp}\left(\mathbf{a}\left(\mathbf{b}^{m}\right)\right)\right)}{\partial \operatorname{Exp}\left(a_{i}\left(\mathbf{b}^{m}\right)\right)} \cdot \operatorname{ED}\left(a_{i}\left(\mathbf{b}^{m}\right)\right) \\
& +\sum_{i}^{C} \sum_{l}^{C_{1}} \frac{\partial \mathbf{U}_{j}\left(\operatorname{Exp}\left(\mathbf{a}\left(\mathbf{b}^{m}\right)\right)\right)}{\partial \operatorname{Exp}\left(a_{i}\left(\mathbf{b}^{m}\right)\right)} \frac{\partial \operatorname{Exp}\left(a_{i}\left(\mathbf{b}^{m}\right)\right)}{\partial b_{l}^{I}} \Delta \hat{b}_{l, N}^{I} \delta \\
& +\sum_{i}^{C} \sum_{l}^{C_{1}} \frac{\partial \mathbf{U}_{j}\left(\operatorname{Exp}\left(\mathbf{a}\left(\mathbf{b}^{m}\right)\right)\right)}{\partial \operatorname{Exp}\left(a_{i}\left(\mathbf{b}^{m}\right)\right)} \frac{\partial E D\left(a_{i}\left(\mathbf{b}^{m}\right)\right)}{\partial b_{l}^{I}} \Delta \hat{b}_{l, N}^{I} \delta \\
& +\sum_{i}^{C} \sum_{l}^{C_{1}} \frac{\partial^{2} \mathbf{U}_{j}\left(\operatorname{Exp}\left(\mathbf{a}\left(\mathbf{b}^{m}\right)\right)\right)}{\partial \operatorname{Exp}\left(a_{i}\left(\mathbf{b}^{m}\right)\right)^{2}} \frac{\partial \operatorname{Exp}\left(a_{i}\left(\mathbf{b}^{m}\right)\right)}{\partial b_{l}^{I}} \Delta \hat{b}_{l, N}^{I} \delta \cdot \operatorname{ED}\left(a_{i}\left(\mathbf{b}^{m}\right)\right)
\end{aligned}
$$

Consequently, the extreme bounds consisting of lower and upper bounds can be obtained by

$$
\underline{\mathbf{U}}_{j}=\min _{\substack{N=1,2, \ldots, L \\ L=l_{1} l_{2}, \cdots, I_{i n}}}\left(\mathbf{U}_{j, N}\left(\mathbf{a}^{R}\left(\mathbf{b}^{I}\right)\right)\right), \overline{\mathbf{U}}_{j}=\max _{\substack{N=1,2, \ldots, L \\ L=l_{1} \cdot l_{2}, l_{i n}}}\left(\mathbf{U}_{j, N}\left(\mathbf{a}^{R}\left(\mathbf{b}^{I}\right)\right)\right)
$$

\subsubsection{Extreme bounds of the expectations and standard variances of the mode shape}

Through the description of Eq. (55), the expectation of the extreme bounds for the $j$-th mode shape can be obtained as follows:

$$
\begin{aligned}
& \operatorname{Exp}\left(\underline{\mathbf{U}}_{j}\right)=\min _{\substack{N=1,2, \ldots, L \\
L=l_{1}, l_{2}, l_{i n}}}\left\{\mathbf{U}_{j}\left(\operatorname{Exp}\left(\mathbf{a}\left(\mathbf{b}^{m}\right)\right)\right)+\sum_{i}^{C} \sum_{l}^{C_{1}} \frac{\partial \mathbf{U}_{j}\left(\operatorname{Exp}\left(\mathbf{a}\left(\mathbf{b}^{m}\right)\right)\right)}{\partial \operatorname{Exp}\left(a_{i}\left(\mathbf{b}^{m}\right)\right)} \frac{\partial \operatorname{Exp}\left(a_{i}\left(\mathbf{b}^{m}\right)\right)}{\partial b_{l}^{I}} \Delta \hat{b}_{l, N}^{I} \delta\right\} \\
& \operatorname{Exp}\left(\overline{\mathbf{U}}_{j}\right)=\max _{\substack{N=1,2, \ldots, L \\
L=l_{1}, l_{2}, \ldots, l_{i n}}}\left\{\mathbf{U}_{j}\left(\operatorname{Exp}\left(\mathbf{a}\left(\mathbf{b}^{m}\right)\right)\right)+\sum_{i}^{C} \sum_{l}^{C_{1}} \frac{\partial \mathbf{U}_{j}\left(\operatorname{Exp}\left(\mathbf{a}\left(\mathbf{b}^{m}\right)\right)\right)}{\partial \operatorname{Exp}\left(a_{i}\left(\mathbf{b}^{m}\right)\right)} \frac{\partial \operatorname{Exp}\left(a_{i}\left(\mathbf{b}^{m}\right)\right)}{\partial b_{l}^{I}} \Delta \hat{b}_{l, N}^{I} \delta\right\}
\end{aligned}
$$

where $\operatorname{Exp}\left(\underline{\mathbf{U}}_{j}\right)$ and $\operatorname{Exp}\left(\overline{\mathbf{U}}_{j}\right)$ denote the expectations of the lower and upper bounds of the eigenvector, respectively. Following Eq. (41), the bounded expectation for the displacement/pressure mode shape can be written as:

$$
\operatorname{Exp}^{I}\left(\mathbf{U}_{j}\right)=\left[\operatorname{Exp}\left(\underline{\mathbf{U}}_{j}\right), \operatorname{Exp}\left(\overline{\mathbf{U}}_{j}\right)\right]
$$

Similarly, the standard variance of the extreme bounds can be expressed as: 


$$
\begin{aligned}
& \operatorname{ED}\left(\underline{\mathbf{U}}_{j}\right)=\min _{\substack{N=1,2, \ldots, L \\
L=l_{l}, l_{2}, l_{i n}}}\left\{\begin{array}{l}
\sum_{i}^{c} \frac{\partial \mathbf{U}_{j}\left(\operatorname{Exp}\left(\mathbf{a}\left(\mathbf{b}^{m}\right)\right)\right)}{\partial \operatorname{Exp}\left(a_{i}\left(\mathbf{b}^{m}\right)\right)} \cdot \operatorname{ED}\left(a_{i}\left(\mathbf{b}^{m}\right)\right) \\
\sum_{l}^{C} \frac{\partial \mathbf{U}_{j}\left(\operatorname{Exp}\left(\mathbf{a}\left(\mathbf{b}^{m}\right)\right)\right)}{\partial \operatorname{Exp}\left(a_{i}\left(\mathbf{b}^{m}\right)\right)} \frac{\partial E D\left(a_{i}\left(\mathbf{b}^{m}\right)\right)}{\partial b_{l}^{I}} \Delta \hat{b}_{l, N}^{I} \delta \\
+\sum_{i}^{C} \sum_{l}^{C_{1}} \frac{\partial^{2} \mathbf{U}_{j}\left(\operatorname{Exp}\left(\mathbf{a}\left(\mathbf{b}^{m}\right)\right)\right)}{\partial \operatorname{Exp}\left(a_{i}\left(\mathbf{b}^{m}\right)\right)^{2}} \frac{\partial \operatorname{Exp}\left(a_{i}\left(\mathbf{b}^{m}\right)\right)}{\partial b_{l}^{I}} \operatorname{ED}\left(a_{i}\left(\mathbf{b}^{m}\right)\right) \Delta \hat{b}_{l, N}^{I} \delta
\end{array}\right\} \\
& E D\left(\overline{\mathbf{U}}_{j}\right)=\max _{\substack{N=1,2, \ldots, L \\
L=l_{l}, l_{2}, l_{l_{n}}}}\left\{\begin{array}{l}
\sum_{i}^{c} \frac{\partial \mathbf{U}_{j}\left(\operatorname{Exp}\left(\mathbf{a}\left(\mathbf{b}^{m}\right)\right)\right)}{\partial \operatorname{Exp}\left(a_{i}\left(\mathbf{b}^{m}\right)\right)} \cdot \operatorname{ED}\left(a_{i}\left(\mathbf{b}^{m}\right)\right) \\
+\sum_{l}^{C} \frac{\partial \mathbf{U}_{j}\left(\operatorname{Exp}\left(\mathbf{a}\left(\mathbf{b}^{m}\right)\right)\right)}{\partial \operatorname{Exp}\left(a_{i}\left(\mathbf{b}^{m}\right)\right)} \frac{\partial E D\left(a_{i}\left(\mathbf{b}^{m}\right)\right)}{\partial b_{l}^{I}} \Delta \hat{b}_{l, N}^{I} \delta \\
+\sum_{i}^{C} \sum_{l}^{C_{1}} \frac{\partial^{2} \mathbf{U}_{j}\left(\operatorname{Exp}\left(\mathbf{a}\left(\mathbf{b}^{m}\right)\right)\right)}{\partial \operatorname{Exp}\left(a_{i}\left(\mathbf{b}^{m}\right)\right)^{2}} \frac{\partial \operatorname{Exp}\left(a_{i}\left(\mathbf{b}^{m}\right)\right)}{\partial b_{l}^{I}} \operatorname{ED}\left(a_{i}\left(\mathbf{b}^{m}\right)\right) \Delta \hat{b}_{l, N}^{I} \delta
\end{array}\right\}
\end{aligned}
$$

where $E D\left(\underline{\mathbf{U}}_{j}\right)$ and $E D\left(\overline{\mathbf{U}}_{j}\right)$ represent the standard variance of the intervals of the eigenvector. Then the extreme bounds of the standard variance are

$$
E D^{I}\left(\mathbf{U}_{j}\right)=\left[E D\left(\underline{\mathbf{U}}_{j}\right), E D\left(\overline{\mathbf{U}}_{j}\right)\right]
$$

Based on Eqs. (57) and (59), the random property and interval property of the $j$-th eigenvector can be all quantified by the expectation bounds and the standard variance bounds of the eigenvector.

\section{Numerical examples}

The influences of the hybrid uncertainty on the physical responses (band structures and pressure/displacement of mode shape) are investigated through several numerical examples, where different materials combinations (fluid matrix or solid matrix) and lattice forms are considered. In this section, without loss of generality, the materials used to simulate the fluid/solid or solid/fluid PCs are aluminum and water, where their 
material properties are listed in Tab. 1. It is noted that the random type in the numerical examples is assumed as the normal distribution, while its expectation locates in an assumed interval and the standard variance of the normal distribution is given with a constant.

Tab. 1 Characteristics of the interval random parameters

\begin{tabular}{ccccc}
\hline \multicolumn{2}{c}{$\begin{array}{c}\text { Interval random variables } \\
\text { Young's } \\
\text { modulus }\end{array}$} & $\begin{array}{c}\text { Distribution } \\
\text { type }\end{array}$ & \multicolumn{2}{c}{ Distribution parameters } \\
$\begin{array}{c}\text { Solid mass } \\
\text { density }\end{array}$ & $\rho_{s}\left(\mathrm{~kg} / \mathrm{m}^{3}\right)$ & Normal & $\operatorname{Exp}\left(\rho_{s}\right) \in 2700\left[1-\delta_{s}, 1+\delta_{s}\right]$ & $E D\left(\rho_{s}\right)=135$ \\
$\begin{array}{c}\text { Poisson's } \\
\text { ratio }\end{array}$ & $v$ & Normal & $\operatorname{Exp}(v) \in 0.3\left[1-\delta_{s}, 1+\delta_{s}\right]$ & $E D(v)=0.015$ \\
$\begin{array}{c}\text { Fluid } \\
\text { velocity }\end{array}$ & $c(\mathrm{~m} / \mathrm{s})$ & Normal & $\operatorname{Exp}(c) \in 1500\left[1-\delta_{f}, 1+\delta_{f}\right]$ & $E D(c)=75$ \\
$\begin{array}{c}\text { Fluid mass } \\
\text { density }\end{array}$ & $\rho_{f}\left(\mathrm{~kg} / \mathrm{m}^{3}\right)$ & Normal & $\operatorname{Exp}\left(\rho_{f}\right) \in 1000\left[1-\delta_{f}, 1+\delta_{f}\right]$ & $E D\left(\rho_{f}\right)=50$ \\
\hline
\end{tabular}

where $\delta_{s}$ and $\delta_{f}$ are the interval uncertainty level for the solid and fluid respectively. It should be noted that the five parameters are assumed to be independent of each other.

Therefore, the results derived from the MCS with 100,000 samples are considered as the reference solutions, where the sample size of interval variables is 100 and the sample size of random parameters is 1000 . The Monte Carlo simulation (MCS) that acts as a powerful tool to obtain the reference solution for the random analysis is extensively applied due to its probability convergence feature. Then, all the predetermined combinations of the uncertain parameters form the part of Monte-Carlo loop which is used to compute the extreme bounds of the band diagrams. Meanwhile, the band structures are figured out, as the assembling of the mass and stiffness matrices is finished and the sweeping along the boundary of the irreducible Brillion zone is implemented. In our simulation, computational efficiency is also validated 
based on the CPU consumed time (MATLAB 2014a, Processor: Intel ${ }^{\circledR}$ Xeon $^{\circledR}$ E5-2630v3 @ 2.4GHz, 16.00GB RAM). On the other hand, the proposed HUMR-FEM is also implemented using the MATLAB codes. In specific, the mass-redistributed operation is realized in the assemble process of the mass matrix, this is same as the traditional FEM process, which can be seen in Ref. [45]. The hybrid uncertainty analysis is then conducted through the MATLAB codes.

\subsection{Solid/fluid PCs with square lattice}

In this subsection, we firstly consider the solid/fluid PCs with the square lattice, as shown in Fig. 4, where the matrix is water, the lattice constant is $a=0.15 \mathrm{~m}$ and the radius of the aluminum scatterers is $r=0.05 \mathrm{~m}$. The solid model is discretized with 705 triangular elements and 385 nodes and the fluid domain is discretized with 696 nodes and 1209 triangular elements.

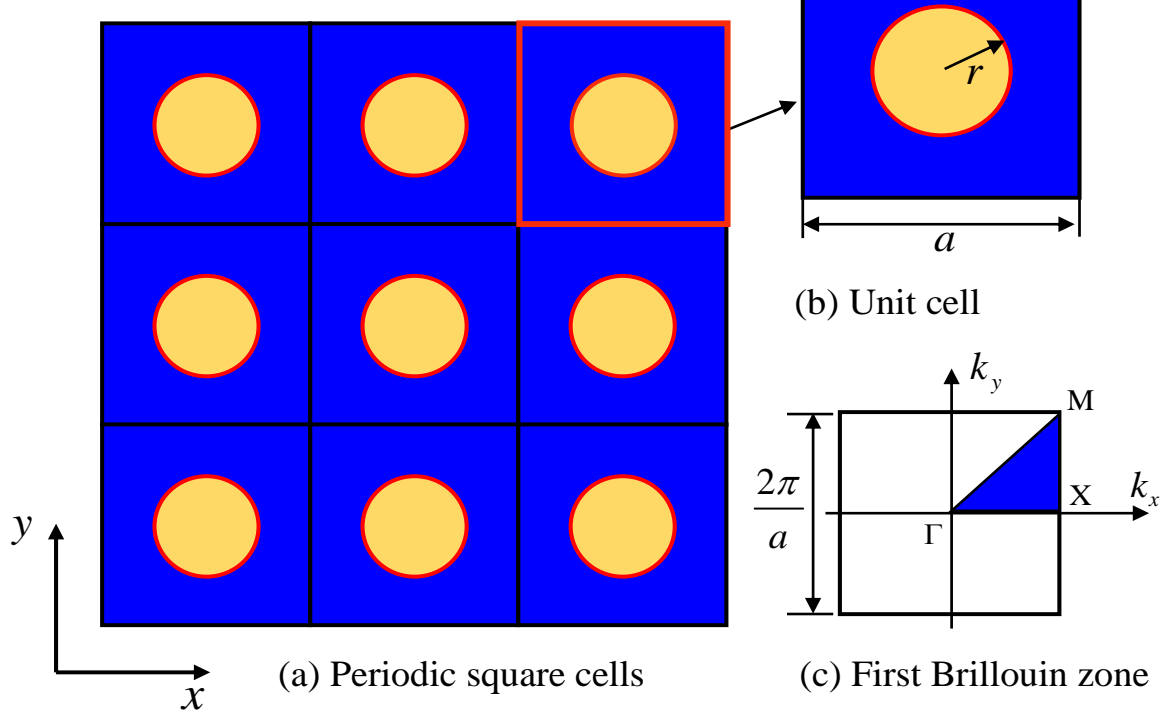

Fig. 4 Solid/fluid PCs with square lattice (The blue and yellow areas denote fluid and solid, respectively)

We firstly consider the small uncertainty level of the expectations of the materials parameters for both the solid and fluid parts, i.e. the corresponding uncertain levels 
are $\delta_{s}=2 \%$ and $\delta_{f}=2 \%$. The fourth to sixth band structures (the first three are rigid modes) are calculated through the path $\Gamma \rightarrow \mathrm{X} \rightarrow \mathrm{M} \rightarrow \Gamma$, and the frequency is presented in the dimensionless unit $\tilde{f}=\omega a / 2 \pi c$ ( $c$ is the sound speed in the fluid). As shown in Fig. 5, the expectation and the standard variance of the band structures are determined by the proposed HUMR-FEM, with LB and UB denoting lower and upper bounds. For the purpose of comparison, the results computed through the MCS are also plotted, with LB MCS and UB MCS denoting lower and upper bounds yielded by MCS. It can be seen that the lower and upper bounds of the expectation and the standard variance obtained by HUMR-FEM are very close to those from the MCS model, indicating that the proposed method is very effective to predict the extreme bounds of the band structures of solid/fluid PCs with uncertainty.

From the physical point of view, there is a stopband around $\tilde{f}=0.5$ along the $\Gamma \mathrm{X}$ direction. As the hybrid uncertainty is introduced to the solid/fluid PCs with FSI, the expected interval of the frequency range of the stopband decreases, as both the upper and lower edges of the bandgaps move toward the center. Besides, since the materials properties of the fluid and solid are both considered to have the interval random uncertainty, the standard variances are no longer a definite constant but a variable interval along the wave propagating vector. To further investigate the effect of the uncertainty level on the band diagrams, we increase the uncertainty level of the materials properties, i.e. from $2 \%$ to $10 \%$ for both solid and fluid domains, and formulate twelve different combinations of uncertain levels listed in Tab. 2. It is found that our method is also effective when the uncertain level reaches $10 \%$, as good 
matching is seen in Fig. 6. The uncertain level of the expectation of the $j$-th band curve defined as $\psi=\left(\bar{\varsigma}_{j}-\underline{\varsigma}_{j}\right) / 2 \varsigma_{j}$ is calculated by the HUMR-FEM, the results of $\Gamma$, $\mathrm{X}$, and $\mathrm{M}$ points are then listed in Tab. 2. Compared Types 1-5 with 6-10, it implies that the uncertainty levels of the expectations of the band diagrams are mainly attributed to the materials uncertainty from the fluid, which also can be seen from the comparison between the Types 1,11 and 3, 12, revealing that these bands are more sensitive to the fluid domain. Moreover, the slopes of the $Г X$ and $Г M$ directions representing the same speed of the fluid also validate that these three modes correspond to the dispersion curves of the fluid domain, indicating that the behavior of these three bands are mainly determined by the motion of the fluid phase. In addition, it is demonstrated from Types 1-5 that the uncertainty levels of the expectations in the band diagrams are monotonous increasing functions with respect to the uncertain levels, when the uncertain levels increase from $2 \%$ to $10 \%$. This means the slight of the fluid properties may result in a discrepancy (near 10\%) between the design and the practical circumstance.

Since the hybrid uncertainty is introduced to the solid/fluid PCs, the pressure contour for the sixth mode shape ( $\Gamma$ point) of the fluid domain is also a random model where the expectations and the standard variances fall in an interval, here we only show the pressure contours for the sixth mode shape ( $\Gamma$ point) by using different methods, as outlined in Figs. 7 and 8. It can be seen that the extreme bounds of the expectation and standard variance of the pressure contour can be well estimated by the HUMR-FEM, as a good agreement between the results obtained from the 
HUMR-FEM and those derived from the MCS is observed.

In order to compare the efficiency, the computational time needed in the two approaches to compute the expectation and standard variances bounds is listed in Tab. 3, showing that the HUMR-FEM is much more efficient than the MCS.

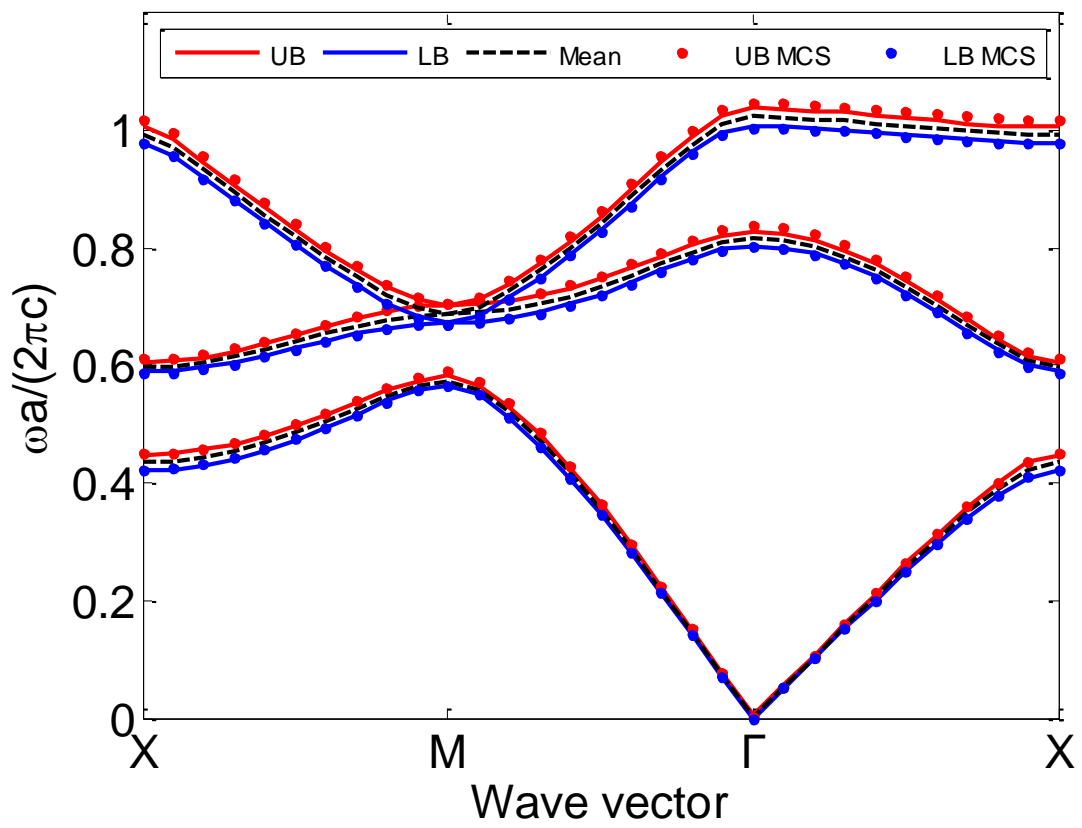

(a)

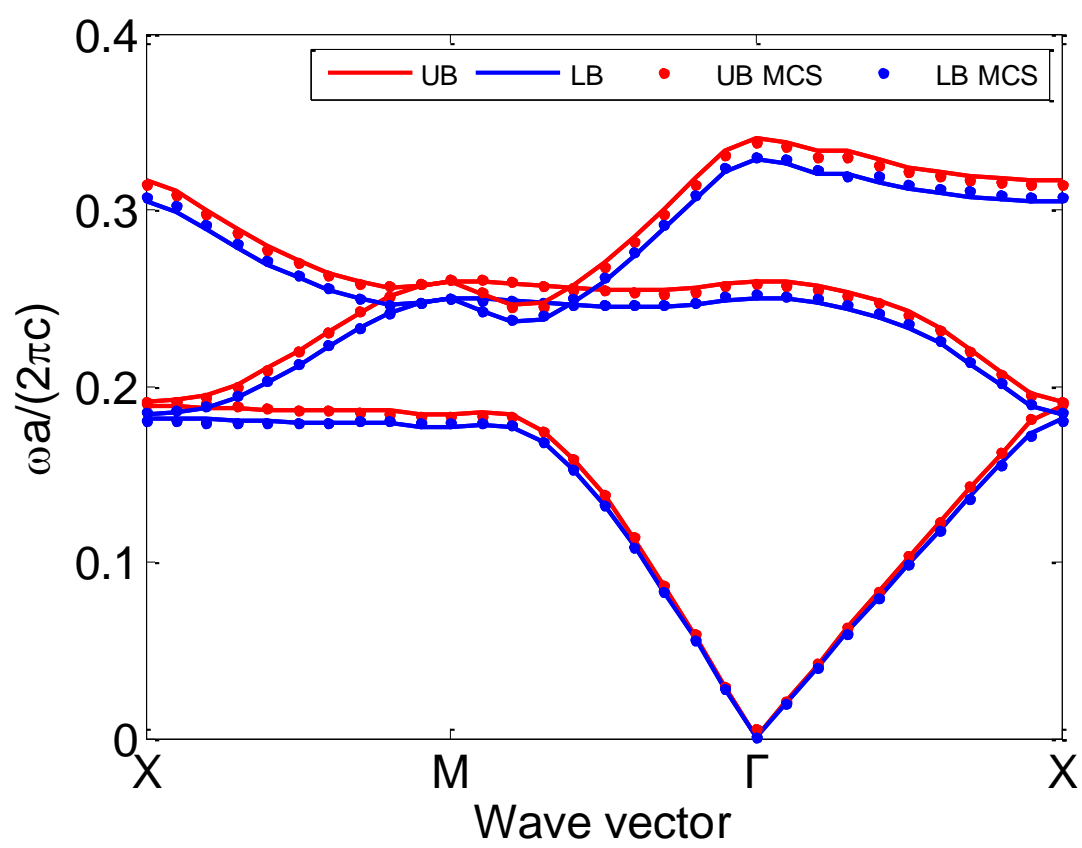

(b) 
Fig. 5 The comparison of the expectations and the standard variance of the band diagrams computed through HUMR-FEM and MCS when the uncertain level is 2\%: (a) expectation; (b) standard variance

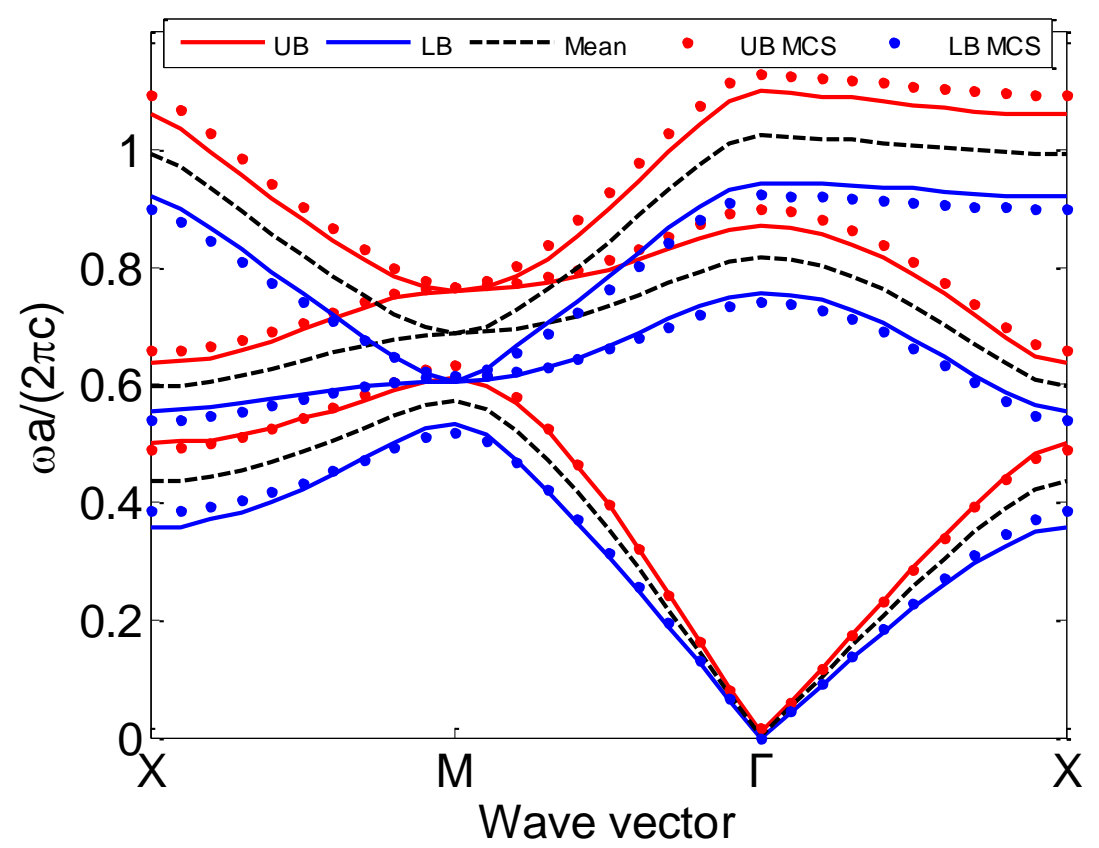

Fig. 6 The comparison of the expectations of the eigenvalues computed through HUMR-FEM and MCS when uncertain level is $10 \%$

Tab. 2 Uncertainty level of the expectation of the sixth band curve

\begin{tabular}{cccccc}
\hline \multicolumn{5}{c}{ Uncertain level of the parameter $(\%)$} & \multicolumn{3}{c}{ Uncertain level $(\%)$} \\
\hline Type & Solid part $\left(\delta_{s}\right)$ & Fluid part $\left(\delta_{f}\right)$ & $\Gamma$ point & X point & M point \\
\hline 1 & 0.0 & 2.0 & 1.46 & 1.34 & 2.03 \\
2 & 0.0 & 4.0 & 2.92 & 2.48 & 4.06 \\
3 & 0.0 & 6.0 & 4.38 & 4.02 & 6.10 \\
4 & 0.0 & 8.0 & 5.85 & 5.37 & 8.14 \\
5 & 0.0 & 10.0 & 7.32 & 6.72 & 10.20 \\
6 & 2.0 & 0.0 & 0.07 & 0.06 & 0.21 \\
7 & 4.0 & 0.0 & 0.14 & 0.13 & 0.43 \\
8 & 6.0 & 0.0 & 0.20 & 0.19 & 0.64 \\
9 & 8.0 & 0.0 & 0.27 & 0.25 & 0.86 \\
10 & 10.0 & 0.0 & 0.34 & 0.31 & 1.07 \\
11 & 2.0 & 2.0 & 1.53 & 1.40 & 2.24 \\
12 & 6.0 & 6.0 & 4.59 & 4.21 & 6.75 \\
\hline
\end{tabular}




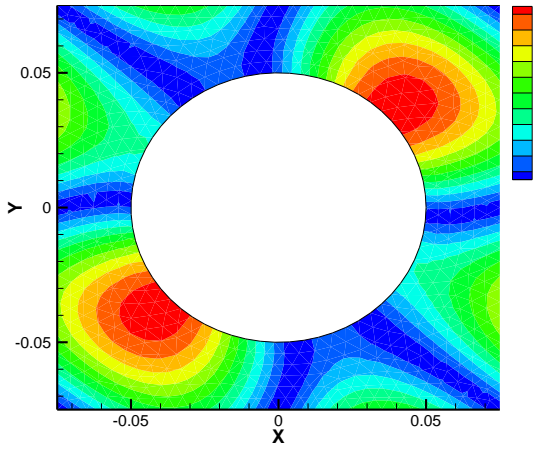

(a) Lower bound using HUMR-FEM

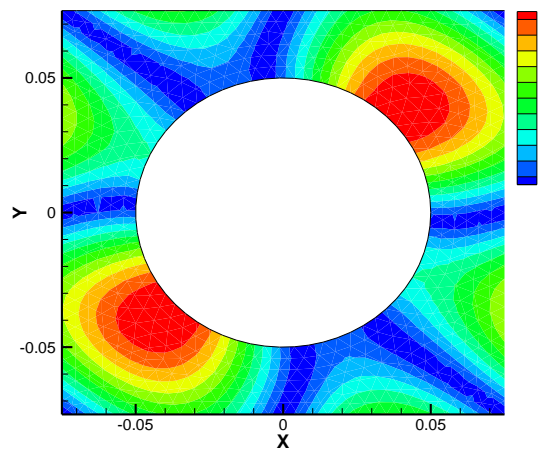

(c) Lower bound using MCS

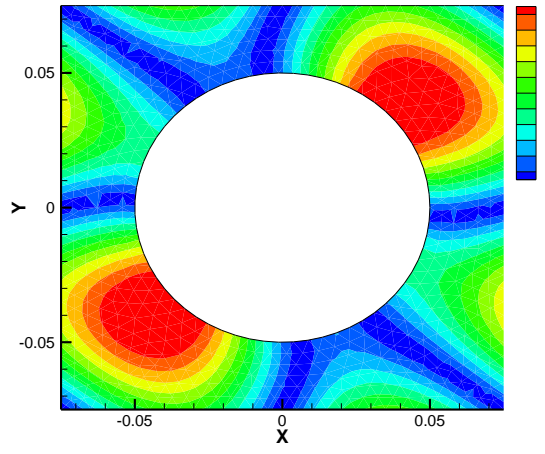

(b) Upper bound using HUMR-FEM

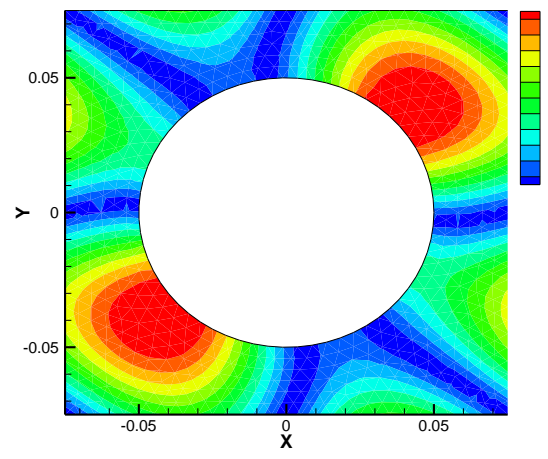

(d) Upper bound using MCS

Fig. 7 The comparison of the expectations of the pressure contour for the sixth mode shape computed through different methods when uncertain level is $2 \%$; (a), (b): Lower and upper bounds using HUMR-FEM; (c), (d): Lower and upper bounds using MCS.

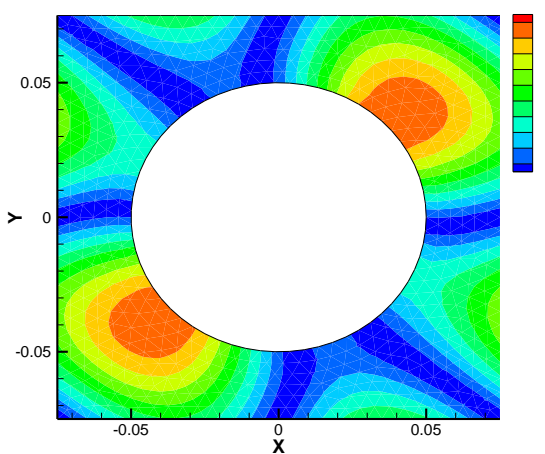

(a) Lower bound using HUMR-FEM

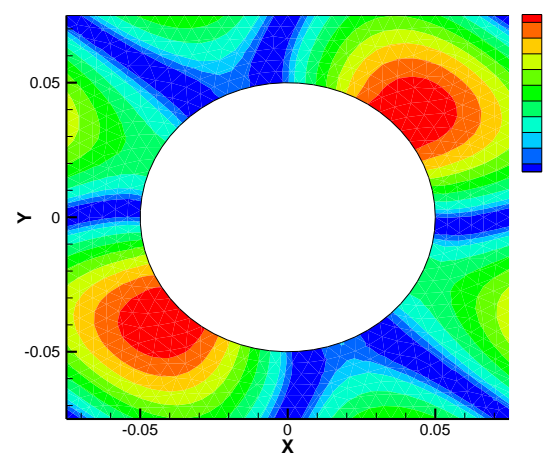

(b) Upper bound using HUMR-FEM 


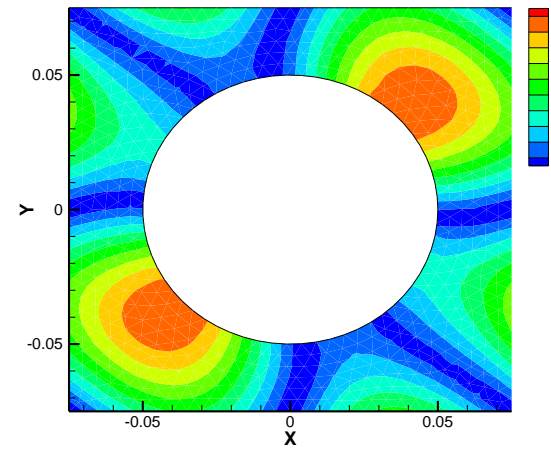

(c) Lower bound using MCS

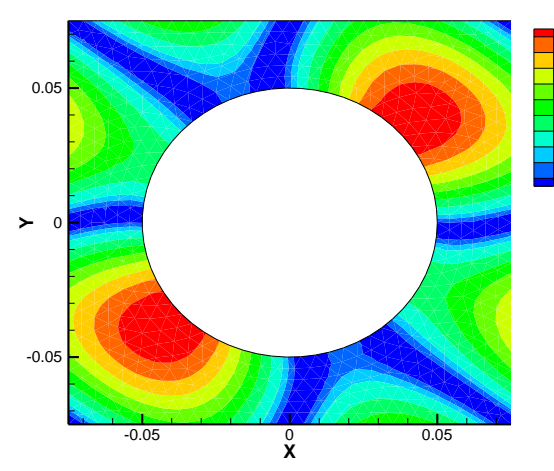

(d) Upper bound using MCS

Fig. 8 The comparison of the standard variance of the pressure contour for the sixth mode shape computed through different methods when the uncertain level is $2 \%$; (a), (b): Lower and upper bounds using HUMR-FEM; (c), (d): Lower and upper bounds using MCS.

Tab.3 Comparison of time needed in different methods (Unit: second)

\begin{tabular}{cccc}
\hline Physical responses & Statistic Property & HUMR-FEM & Monte-Carlo simulation \\
\hline \multirow{2}{*}{ Band structures } & Expectation & 75.5 & 85975 \\
& Standard variance & 183.5 & 71083.6 \\
\hline \multirow{2}{*}{ Pressure contours } & Expectation & 100.0 & 90547.0 \\
& Standard variance & 154.1 & 89378.1 \\
\hline
\end{tabular}

\subsection{Fluid/solid PCs with square lattice}

The fluid/solid PCs with square lattice expressed in Fig. 9 is investigated. The geometrical parameters are lattice constant $a=0.15 \mathrm{~m}$ and the length of square water scatterers $b=0.1 \mathrm{~m}$. The solid meshes include 628 nodes and 1056 triangular elements, the fluid meshes consist 493 nodes and 904 triangular elements.

Mathematically, the bounds of the expectations and the standard variance of the first three non-rigid band structures are computed and depicted in Fig. 10. The acronyms appeared here and hereafter are the same meaning as the first example. It can be seen that the bounds of the expectation and the standard variance calculated by HUMR-FEM share those bounds values with MCS, which validates the effectiveness of the proposed method in the modeling of fluid/solid PCs with FSI when the solid matrix is applied. Besides, when the materials properties are considered as the interval 
random variables, it is clearly seen that the band structures are also a series of interval random variables, whose expectations and standard variances locate in an interval.

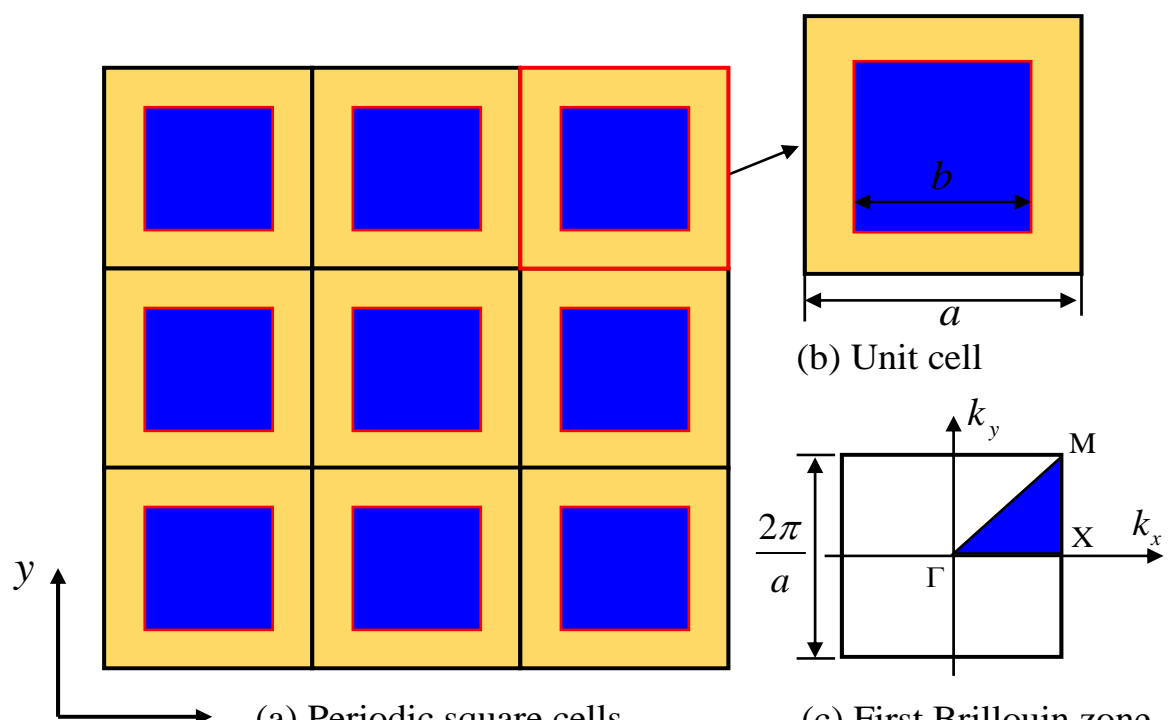

Fig. 9 Fluid/solid PCs with square lattice (The blue and yellow areas are fluid and solid, respectively)

In order to study the effect of uncertain levels on the band diagrams, we increase the uncertainty of the expectations of the materials parameters with twelve combinations and set all the standard variances as an assumed constant (as listed in Tab. 1), as listed in Tab. 4. When the uncertain level is less than $10 \%$, the proposed method is still very effective since the expectation bounds derived through HUMR-FEM agree very well in the lower order band structures in Fig. 11. Moreover, the results in Tab. 4 have shown that the uncertainty of the materials in the fluid dominates the uncertain level of the expectations of the band gap diagrams. This implies that the band gap is more sensitive to the uncertainty of fluid properties compared with solid. Moreover, in the fluid/solid PCs, it can be observed that the uncertain level of the expectations increases monotonously and non-linearly as the uncertainties increase linearly from $2 \%$ to $10 \%$. 
The bounds of the pressure contour for the fifth mode shape ( $\Gamma$ point) are expressed in Figs. 12 and 13. The results obtained from HUMR-FEM agree very well with those derived from MCS, indicating that the proposed method is also very effective in predicting the pressure contour for the mode shapes. In this example, the computational time between the HUMR-FEM and MCS is also compared, as listed in Tab. 5. It can be obviously found that computational efficiency of the HUMU-FEM is much better compared with MCS.

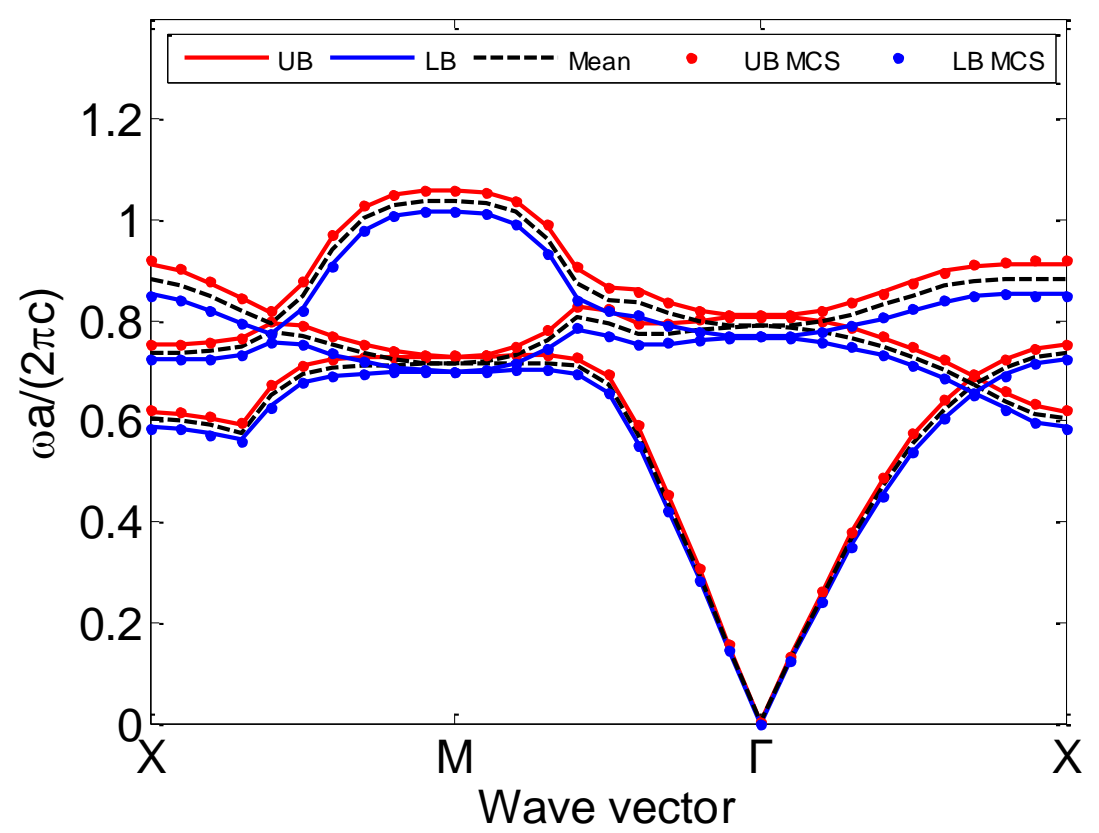

(a) 


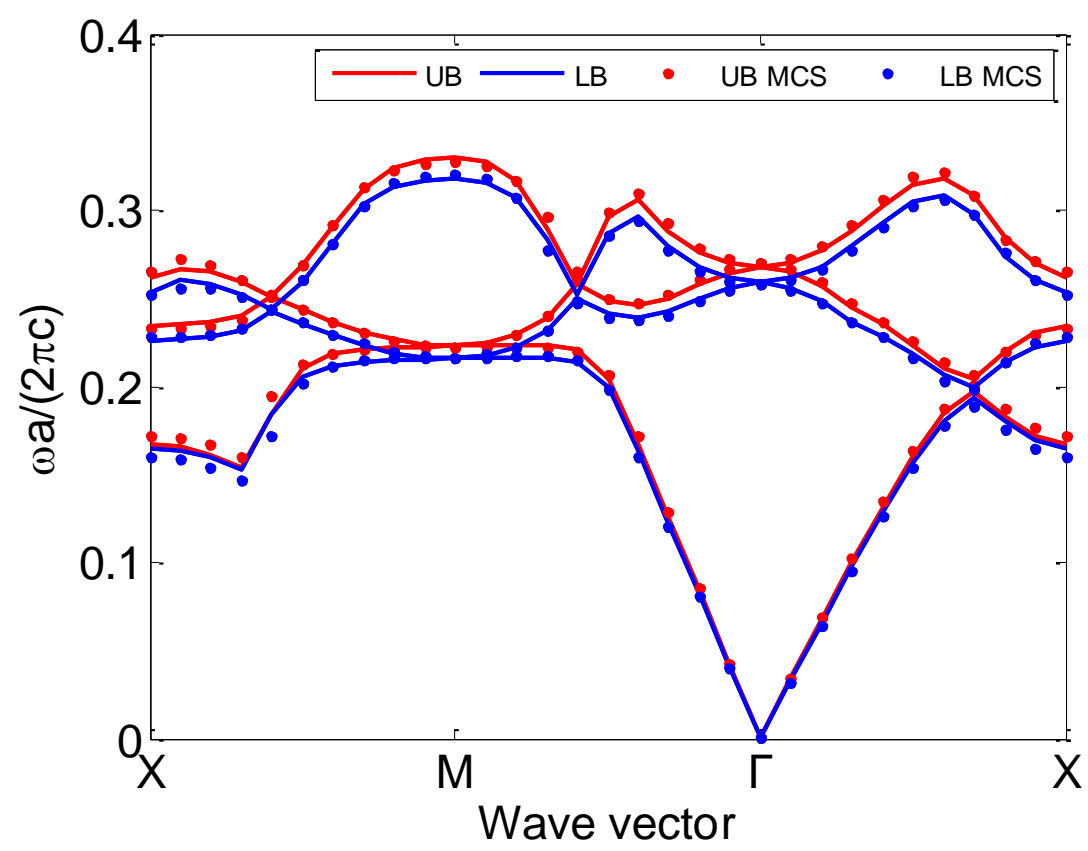

(b)

Fig. 10 The comparison of the expectations and the standard variance of the eigenvalues in fluid/solid PCs with square lattice through different methods when uncertain level is $2 \%$, (a) expectation; (b) standard variance

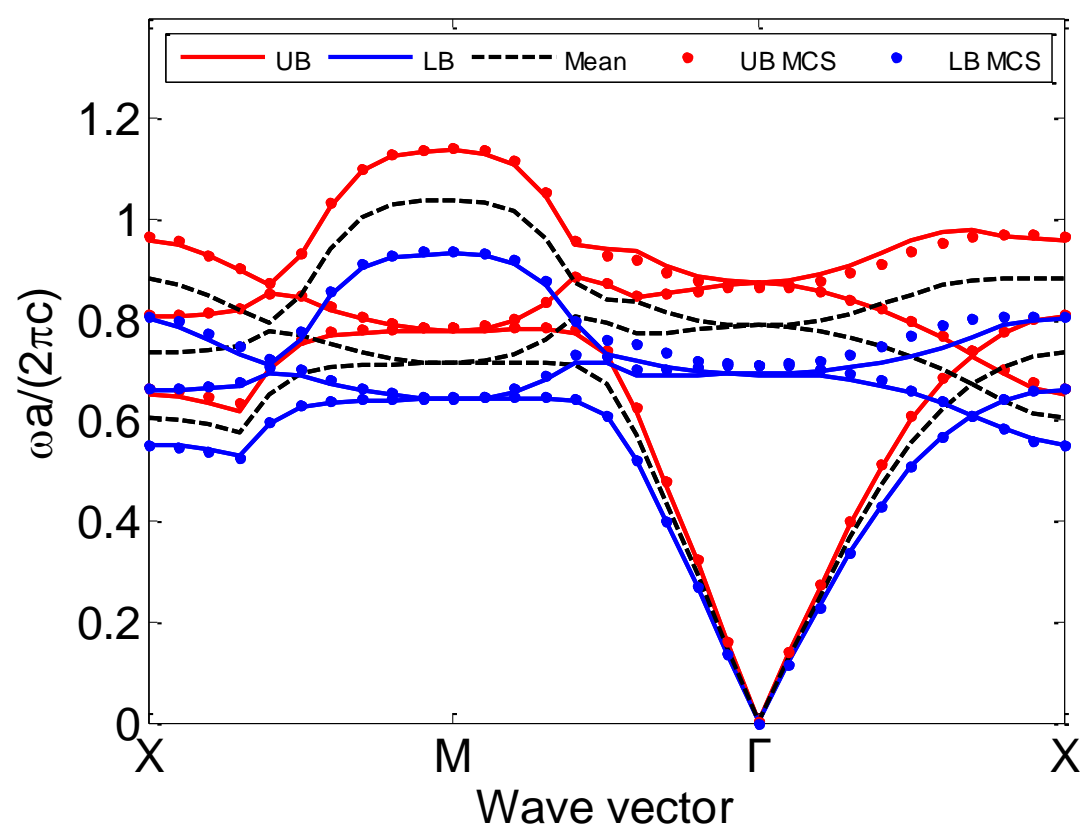

Fig. 11 The comparison of the expectations of the band structures computed through HUMR-FEM and MCS when uncertain level is $10 \%$ 
Tab. 4 Uncertainty level of the expectation of the fifth band curve

\begin{tabular}{cccccc}
\hline \multicolumn{4}{c}{ Uncertain level of the parameter $(\%)$} & \multicolumn{3}{c}{ Uncertain level $(\%)$} \\
\hline Type & Solid part $\left(\delta_{s}\right)$ & Fluid part $\left(\delta_{f}\right)$ & $\Gamma$ point & X point & M point \\
\hline 1 & 0.0 & 2.0 & 1.88 & 2.46 & 1.81 \\
2 & 0.0 & 4.0 & 3.77 & 4.93 & 3.63 \\
3 & 0.0 & 6.0 & 5.66 & 7.40 & 5.45 \\
4 & 0.0 & 8.0 & 7.56 & 9.89 & 7.27 \\
5 & 0.0 & 10.0 & 9.46 & 12.40 & 9.10 \\
6 & 2.0 & 0.0 & 0.19 & 1.17 & 1.31 \\
7 & 4.0 & 0.0 & 0.38 & 2.34 & 2.62 \\
8 & 6.0 & 0.0 & 0.58 & 3.52 & 3.92 \\
9 & 8.0 & 0.0 & 0.77 & 4.69 & 5.24 \\
10 & 10.0 & 0.0 & 0.96 & 5.87 & 6.55 \\
11 & 2.0 & 2.0 & 2.07 & 3.64 & 3.12 \\
12 & 6.0 & 6.0 & 6.24 & 10.97 & 9.4 \\
\hline
\end{tabular}

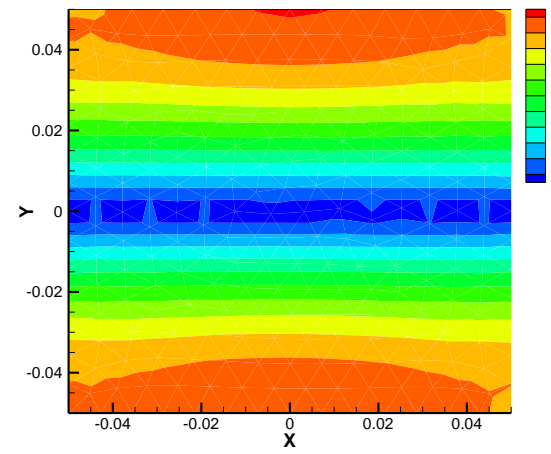

(a) Lower bound using HUMR-FEM

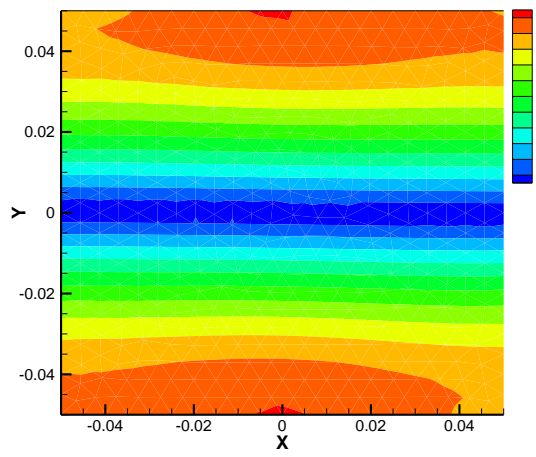

(c) Lower bound using MCS

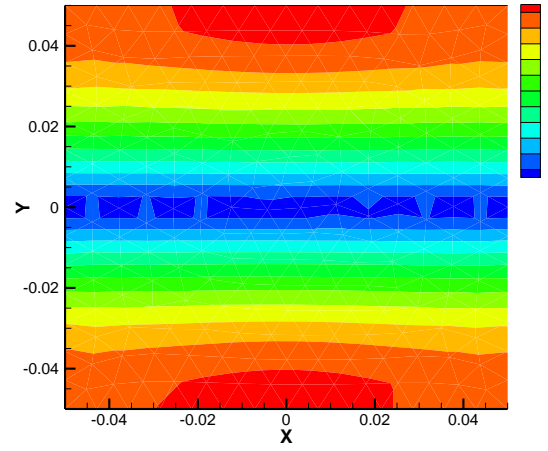

(b) Upper bound using HUMR-FEM

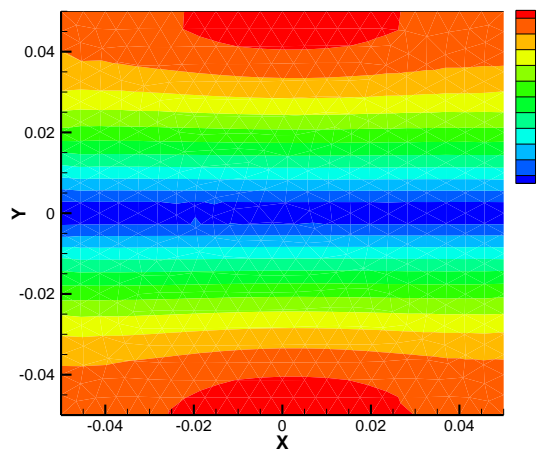

(d) Upper bound using MCS

Fig. 12 The comparison of the expectations of the pressure contour for the fifth mode shape computed through different methods when uncertain level is $2 \%$; (a), (b): Lower and upper bounds using HUMR-FEM; (c), (d): Lower and upper bounds using MCS. 


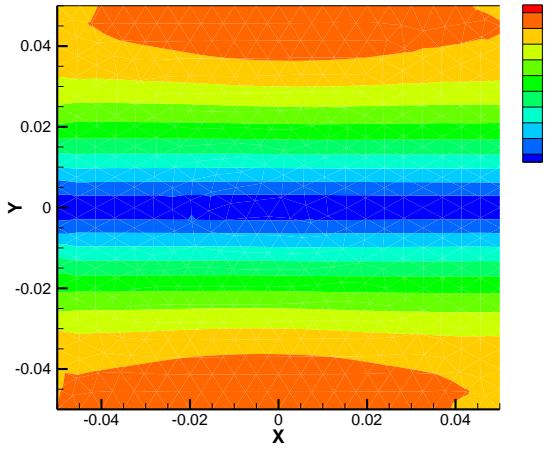

(a) Lower bound using HUMR-FEM

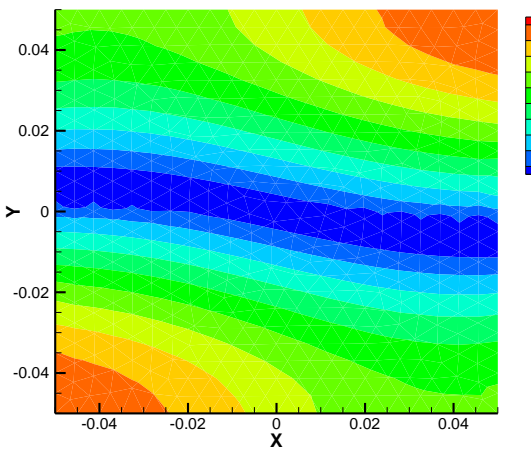

(c) Lower bound using MCS

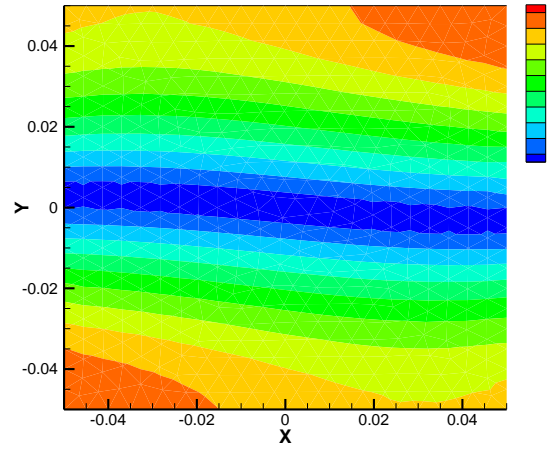

(b) Upper bound using HUMR-FEM

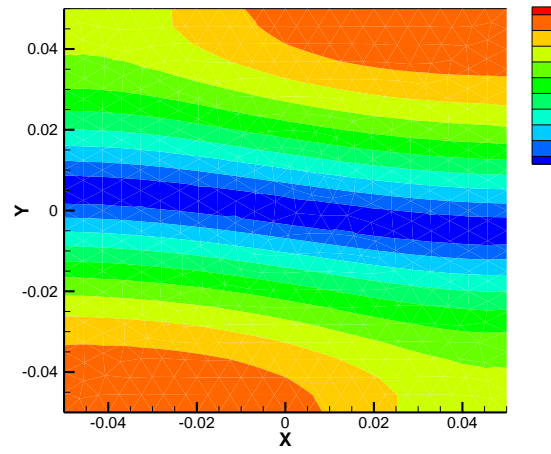

(d) Upper bound using MCS

Fig. 13 The comparison of the standard variance of the pressure contour for the fifth mode shape computed through different methods when uncertain level is $2 \%$; (a), (b): Lower and upper bounds using HUMR-FEM; (c), (d): Lower and upper bounds using MCS.

Tab.5 Time of using different methods (Unit: second)

\begin{tabular}{cccc}
\hline Physical response & Statistic Property & HUMR-FEM & Monte-Carlo Method \\
\hline \multirow{2}{*}{ Band structures } & Expectation & 40.8 & 170794.8 \\
& Standard variance & 313.9 & 65540.0 \\
\hline \multirow{2}{*}{ Pressure contours } & Expectation & 141.7 & 66305.1 \\
& Standard variance & 194.2 & 106913.9 \\
\hline
\end{tabular}

\subsection{Solid/fluid PCs with hexangular lattice}

The hexangular lattice for solid/fluid PCs with FSI expressed in Fig. 14 is studied in this subsection, where the lattice constant is $\mathrm{a}=\sqrt{3} / 20 \mathrm{~m}$ and the aluminum scatterers is $r=0.035 \mathrm{~m}$. The solid mesh includes 385 nodes and 705 triangular elements, while the fluid mesh includes 313 nodes and 479 triangular elements. 


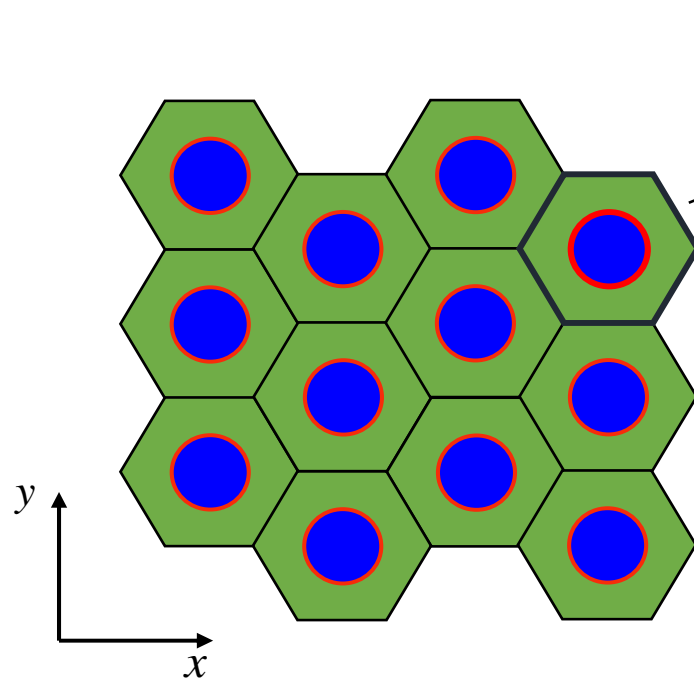

(a) Periodic triangular cells

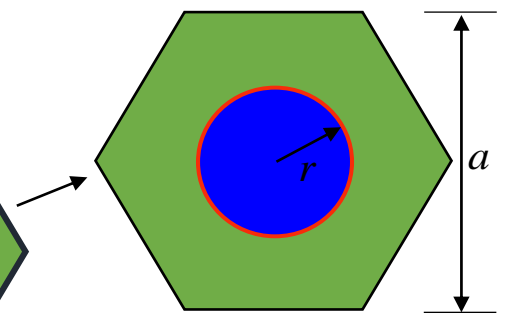

(b) Unit cell

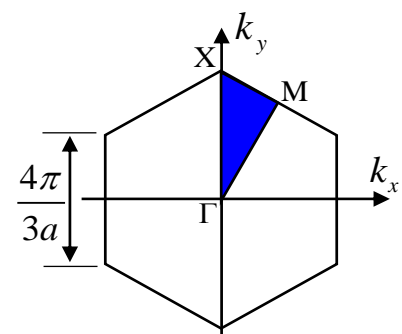

(c) First Brillouin zone

Fig. 14 Solid/fluid PCs with hexangular lattice (The green and blue areas are fluid and solid, respectively)

The expectation bound and the standard variance bound of the band structures are calculated by the proposed method and shown in Fig. 15 when the uncertain level is 2\%. Again, a very good agreement between the HUMR-FEM and MCS is clearly seen. The proposed method is also effective in dealing with the hexangular lattice.

Physically, it can be seen from Fig. 15(a) that the expected interval of the frequency range of the stopband narrows down, as the upper and lower edges of the bandgaps both move toward the central frequency of the bandgap. Since the uncertainties of the solid and the fluid are independent and moreover, the uncertain variables in each domain are also independent, the band diagrams (Fig. 15) are no longer a deterministic value but a random variable with bounded expectations and the standard variances. The influences of uncertainty levels on the band diagrams are investigated, forming twelve types of combination listed in Tab. 6, and the corresponding uncertain levels are computed through HUMR-FEM. The results comparison between the HUMR-FEM and MCS is plotted in Fig. 16 when both the 
uncertain levels of materials properties of the fluid and solid domains are $10 \%$. The results show that the HUMR-FEM gives very similar results obtained from MCS, demonstrating again the HUMR-FEM is reliable. From Tab. 6, it can be obviously observed that the uncertainties of the materials in the fluid domain are the main factors that dictate the uncertainty level of the expectations of the band structures. Besides, it shows that the interval uncertainties of the expectations increase monotonously and non-linearly as the materials uncertainties linearly increase.

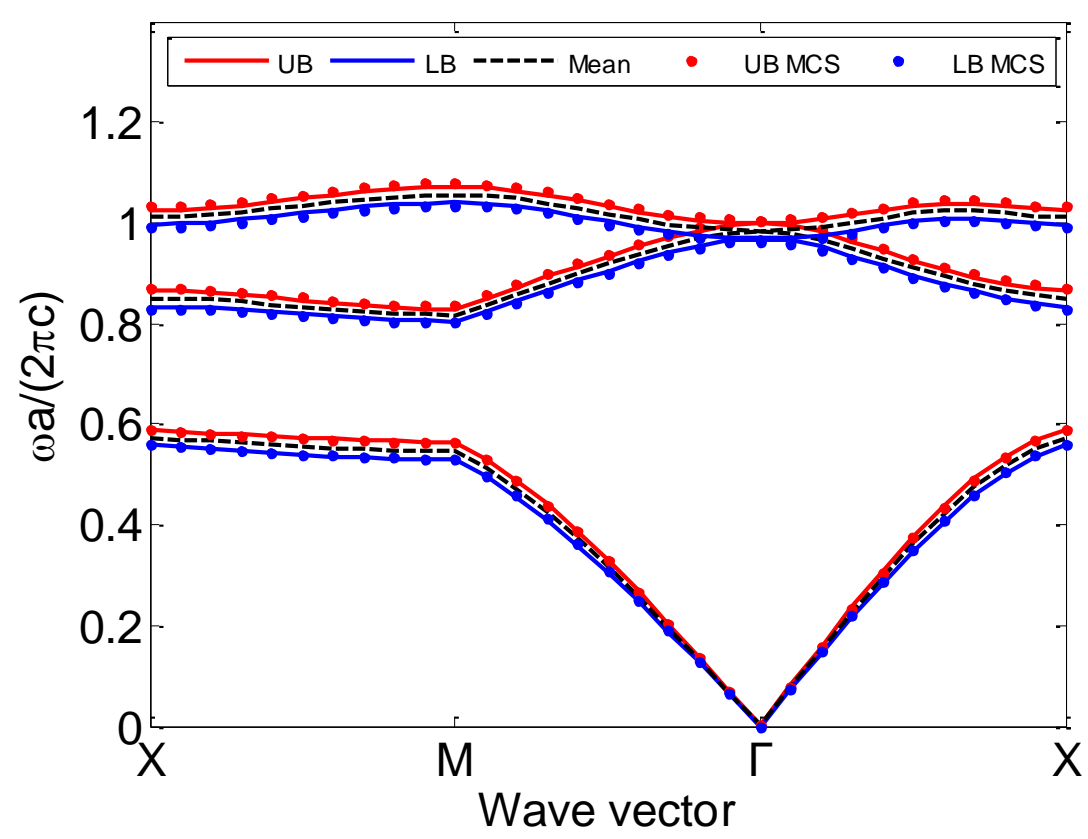

(a) 


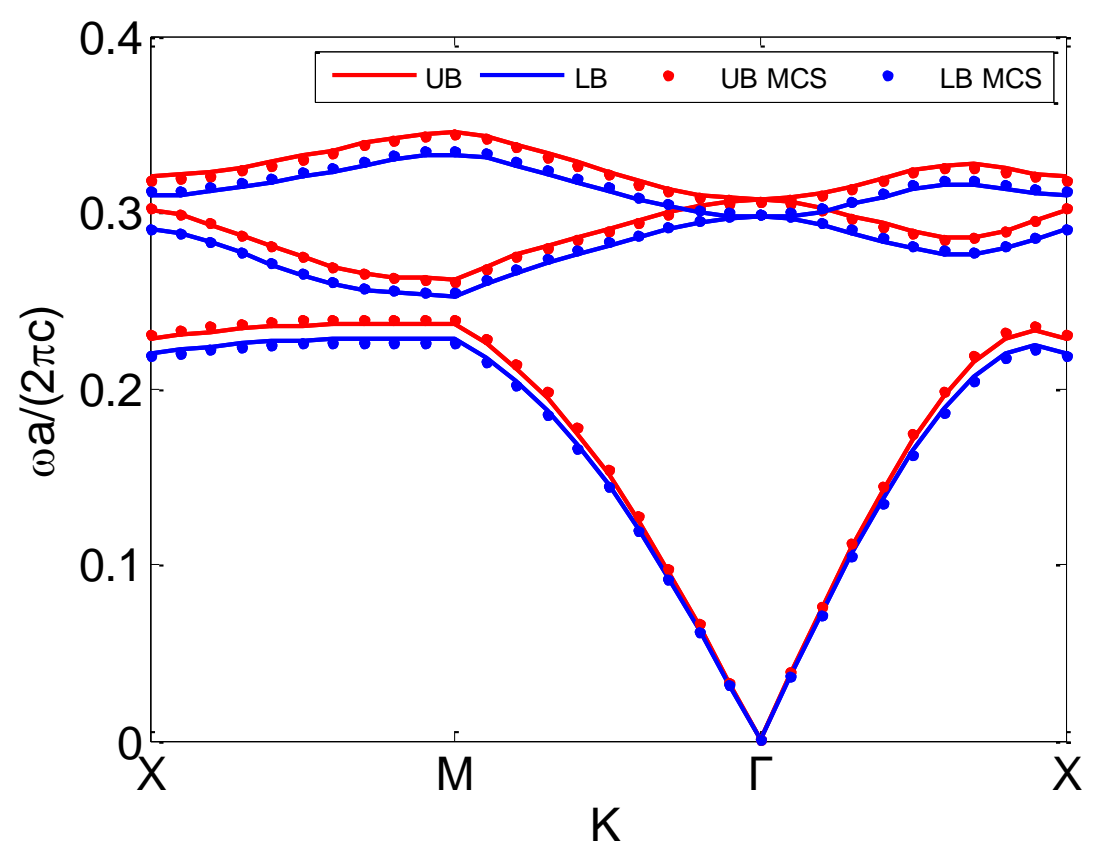

(b)

Fig. 15 The comparison of the expectations and the standard variance of the band structures computed through different methods when the uncertain level is $2 \%$, (a) expectation; (b) standard variance.

Tab. 6 Uncertainty level of the expectation of the fifth band curve

\begin{tabular}{cccccc}
\hline \multicolumn{4}{c}{ Uncertain level of the parameter $(\%)$} & \multicolumn{3}{c}{ Uncertain level $(\%)$} \\
\hline \multirow{2}{*}{ Type } & Solid part $\left(\delta_{s}\right)$ & Fluid part $\left(\delta_{f}\right)$ & $\Gamma$ point & X point & M point \\
\hline 1 & 0.0 & 2.0 & 1.35 & 1.34 & 1.40 \\
2 & 0.0 & 4.0 & 2.71 & 2.69 & 2.78 \\
3 & 0.0 & 6.0 & 4.06 & 4.03 & 4.17 \\
4 & 0.0 & 8.0 & 5.42 & 5.38 & 5.57 \\
5 & 0.0 & 10.0 & 6.76 & 6.73 & 6.96 \\
6 & 2.0 & 0.0 & 0.19 & 0.06 & 0.12 \\
7 & 4.0 & 0.0 & 0.38 & 0.13 & 0.24 \\
8 & 6.0 & 0.0 & 0.57 & 0.19 & 0.35 \\
9 & 8.0 & 0.0 & 0.77 & 0.26 & 0.47 \\
10 & 10.0 & 0.0 & 0.96 & 0.32 & 0.59 \\
11 & 2.0 & 2.0 & 1.54 & 1.41 & 1.51 \\
12 & 6.0 & 6.0 & 4.63 & 4.23 & 4.53 \\
\hline
\end{tabular}




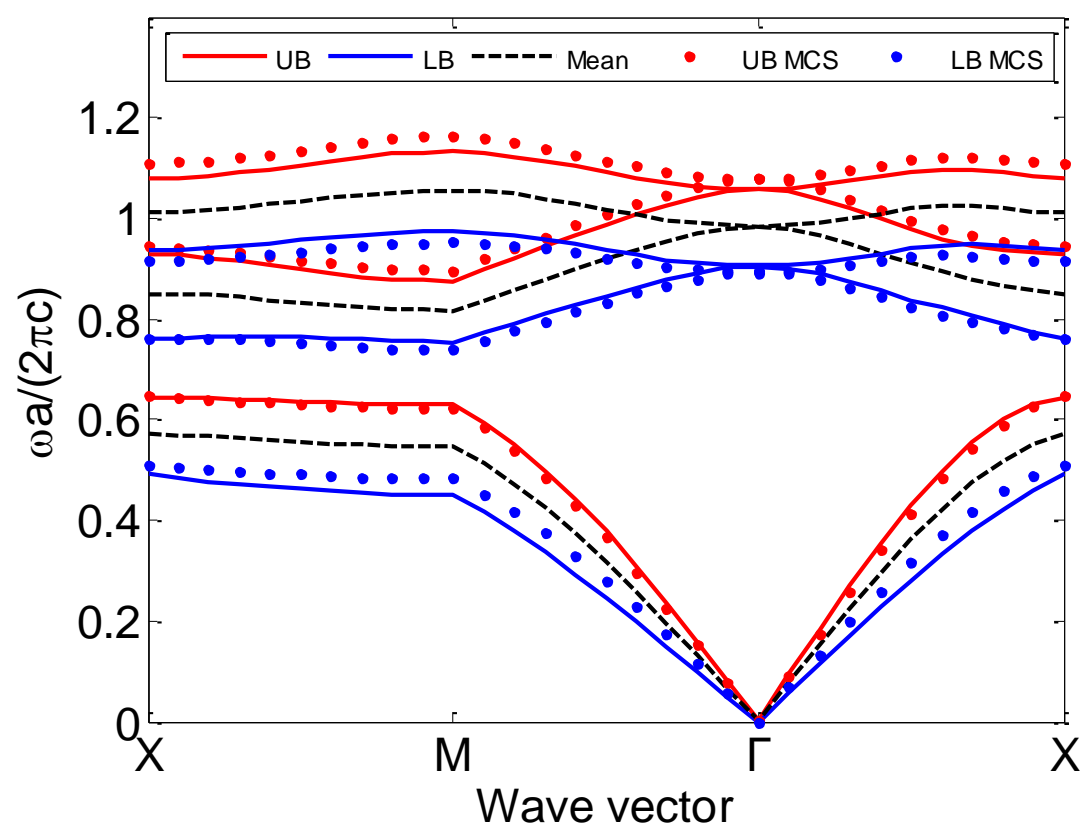

Fig. 16 The comparison of the expectations of the band structures computed through different methods when the uncertain level is $10 \%$

The expectation and standard variance bounds comparison of the pressure contours for the fifth mode shape ( $\Gamma$ point) using HUMR-FEM and MCS is depicted in Figs. 17 and 18. It can be seen that the HUMR-FEM can well predict the bounds of the pressure contour for the fifth pressure mode shape, as the result obtained from the HUMR-FEM matches very well with those yielded by the MCS. The time comparison between the two methods is listed in Tab. 7. Again, it is found that the HUMR-FEM is much more efficient than the MCS.

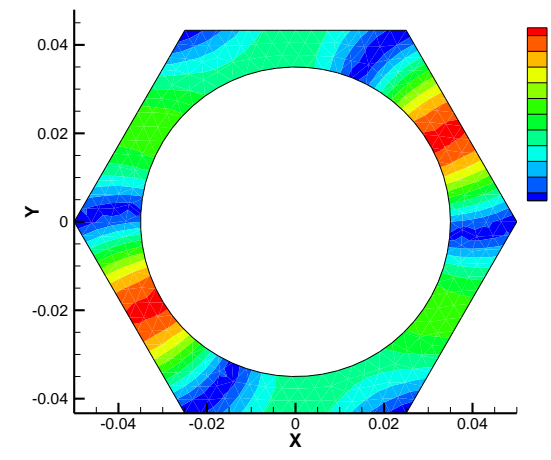

(a) Lower bound using HUMR-FEM

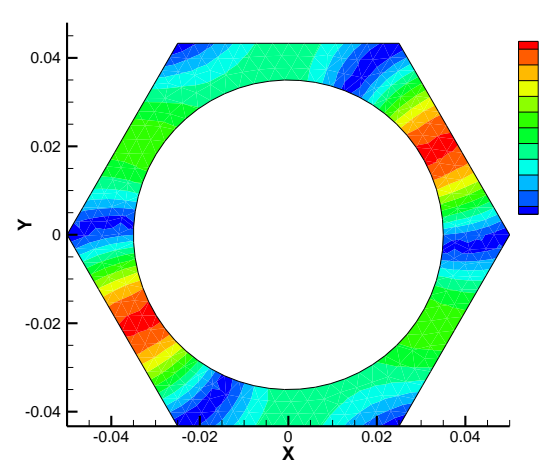

(b) Upper bound using HUMR-FEM 


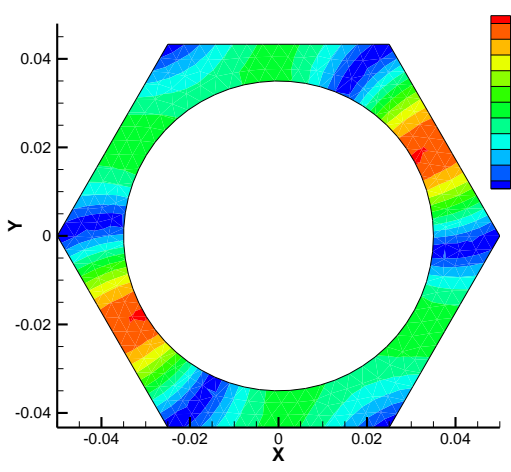

(c) Lower bound using MCS

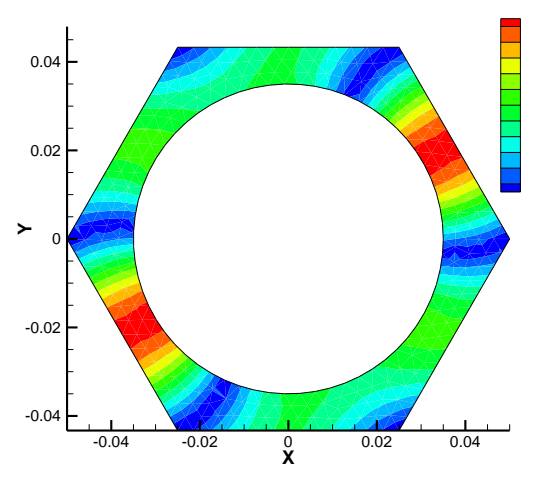

(d) Upper bound using MCS

Fig. 17 The comparison of the expectations of the eigenvectors computed through different methods when uncertain level is 2\%; (a), (b): Lower and upper bounds using HUMR-FEM; (c), (d): Lower and upper bounds using MCS.

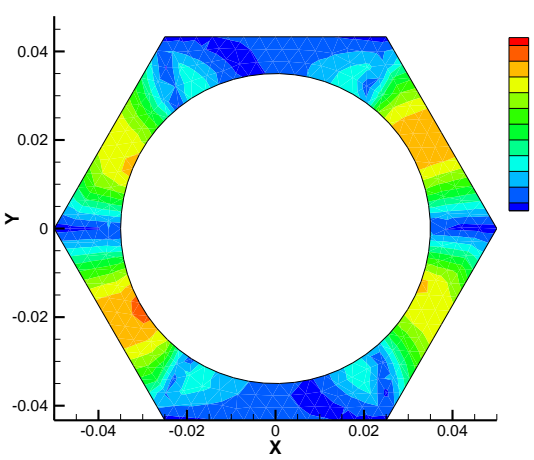

(a) Lower bound using HUMR-FEM

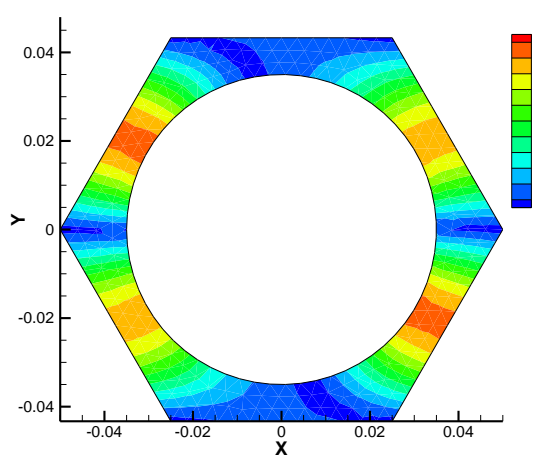

(c) Lower bound using MCS

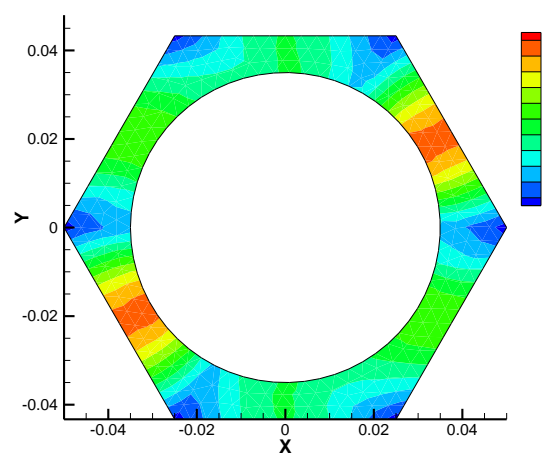

(b) Upper bound using HUMR-FEM

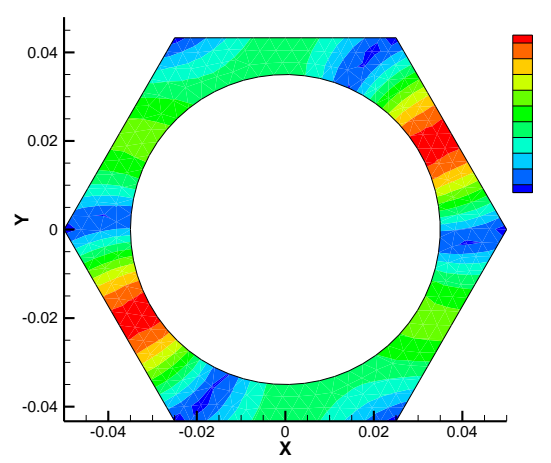

(d) Upper bound using MCS

Fig. 18 The comparison of the standard variance of the eigenvectors computed through different methods when uncertain level is 2\%; (a), (b): Lower and upper bounds using HUMR-FEM; (c), (d): Lower and upper bounds using MCS. 
Tab. 7 Computational time of using different methods (Unit: second)

\begin{tabular}{cccc}
\hline Physical responses & Statistic Property & HUMR-FEM & Monte-Carlo Method \\
\hline \multirow{2}{*}{ Band structures } & Expectation & 34.3 & 21373.8 \\
& Standard variance & 76.6 & 16189.8 \\
\hline \multirow{2}{*}{ Pressure contours } & Expectation & 1269.6 & 63159.5 \\
& Standard variance & 123.4 & 21415.4 \\
\hline
\end{tabular}

\subsection{Fluid/solid PCs with hexangular lattice}

In this subsection, the fluid/solid PCs with FSI and hexangular lattice presented in Fig. 19 is studied, where the lattice constant is $a=\sqrt{3} / 20 \mathrm{~m}$ and the side length of the square scatterers is $b=0.05 \mathrm{~m}$. The unit cell of the current fluid/solid PCs is discretized into 447 nodes with 730 triangular elements for the solid and 244 nodes with 430 triangular elements for the fluid.

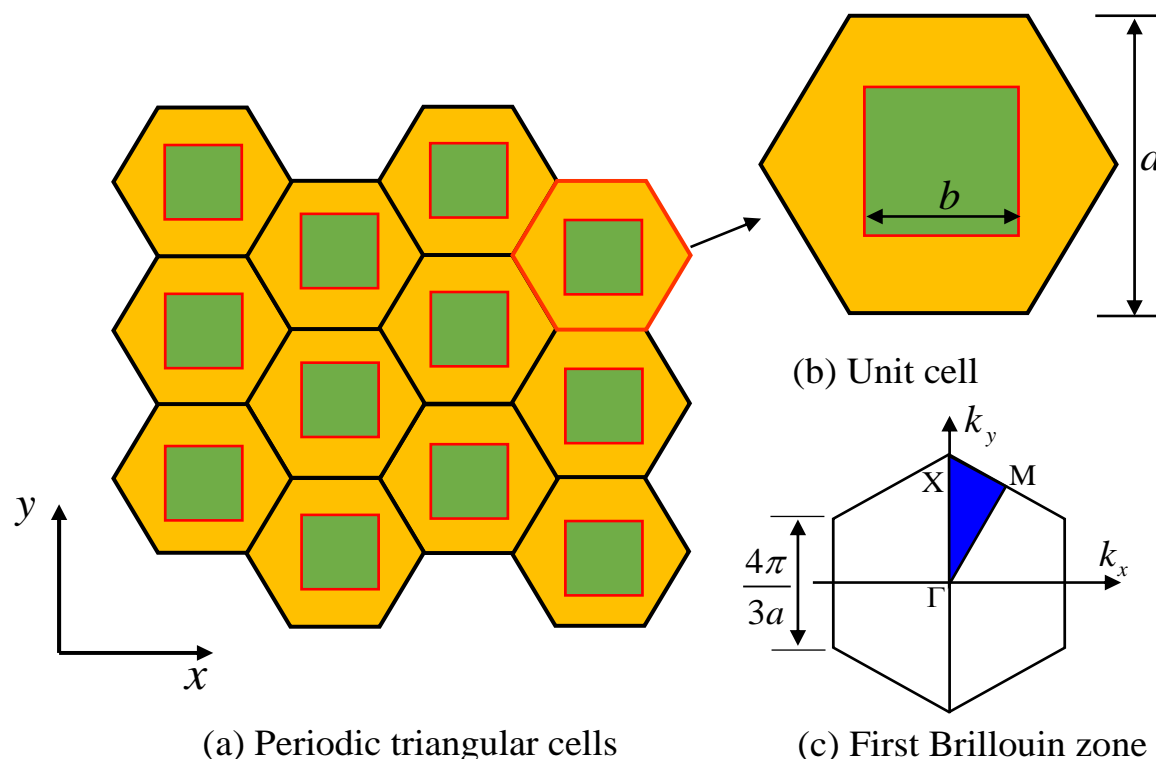

Fig. 19 Fluid/solid PCs with hexangular lattice (The green and orange areas are fluid and solid, respectively)

The extreme bounds of the expectations and standard variance for the band structures computed by the HUMR-FEM and MCS are shown in Fig. 20, when the uncertain level is $2 \%$. It can be found that these bounds derived by the HUMR-FEM agree very well with those obtained by MCS, suggesting that the HUMR-FEM is very effective to predict the physical response of the fluid/solid PCs with uncertainty. Next, 
the effects of the uncertainty level of fluid/solid PC on the band structure are studied using twelve combination levels uncertainty, as listed in Tab. 8. When the uncertainty level is $10 \%$, the comparison between the two methods is depicted in Fig. 21. It can be found that a very good agreement is obtained. When the uncertain level is small, i.e. less than $10 \%$, the expectations and standard variances of the band structures can be well predicted by the proposed HUMR-FEM. From Types 1-5 and 6-10, it is clearly seen that the material property uncertainties of the fluid domain are much more sensitive than those of the solid domain in the expectation computations of the band structures. The uncertain levels of the expectations in types $1-5$ range from $1.35 \%$ to $6.75 \%$, while those in types $6-10$ are no more than $3.1 \%$. This again demonstrates that the change of the fluid properties may be the main factor that affects the uncertainty of the response.

The upper and lower bounds of the pressure contours of the fifth mode shape $(\Gamma$ point) are plotted in Figs. 22 and 23 with the reference solution. Again, it is shown that the expectations and the standard variance bounds can be well predicted under the interval random uncertainty. The computational cost of the two methods is compared and listed in Tab. 9. It shows that the HUMR-FEM can remarkably improve the efficiency compared with the traditional MCS. 


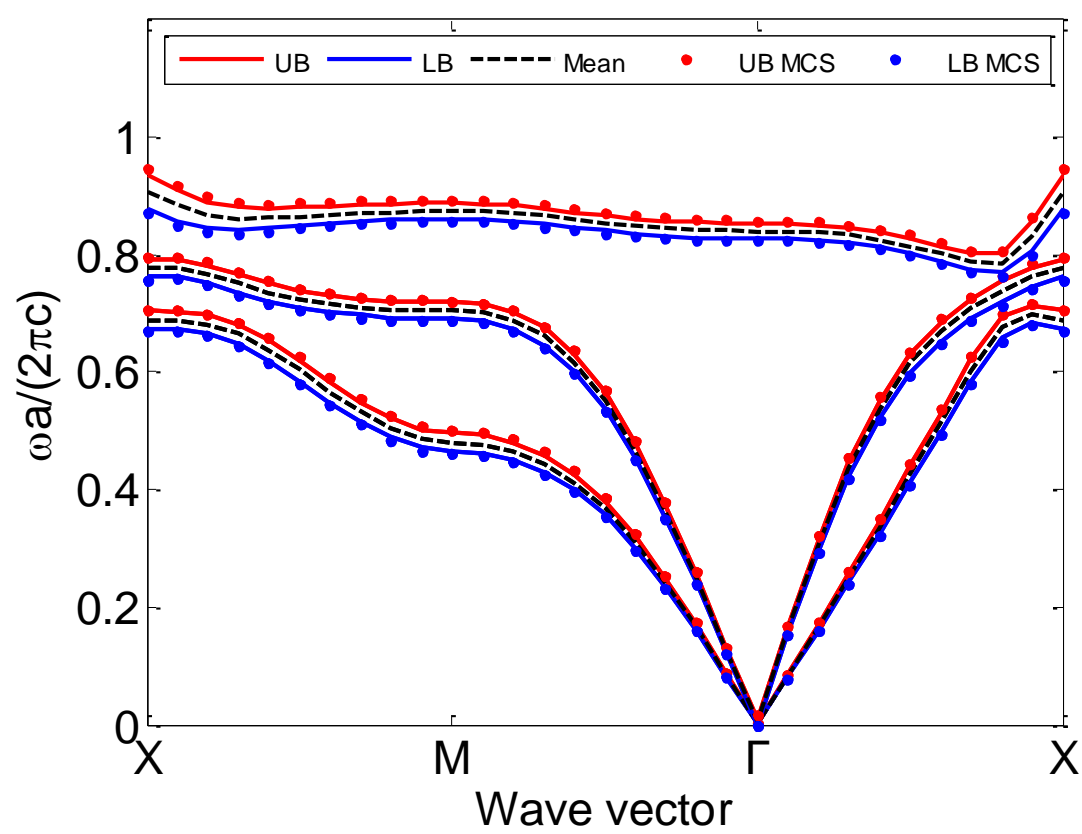

(a)

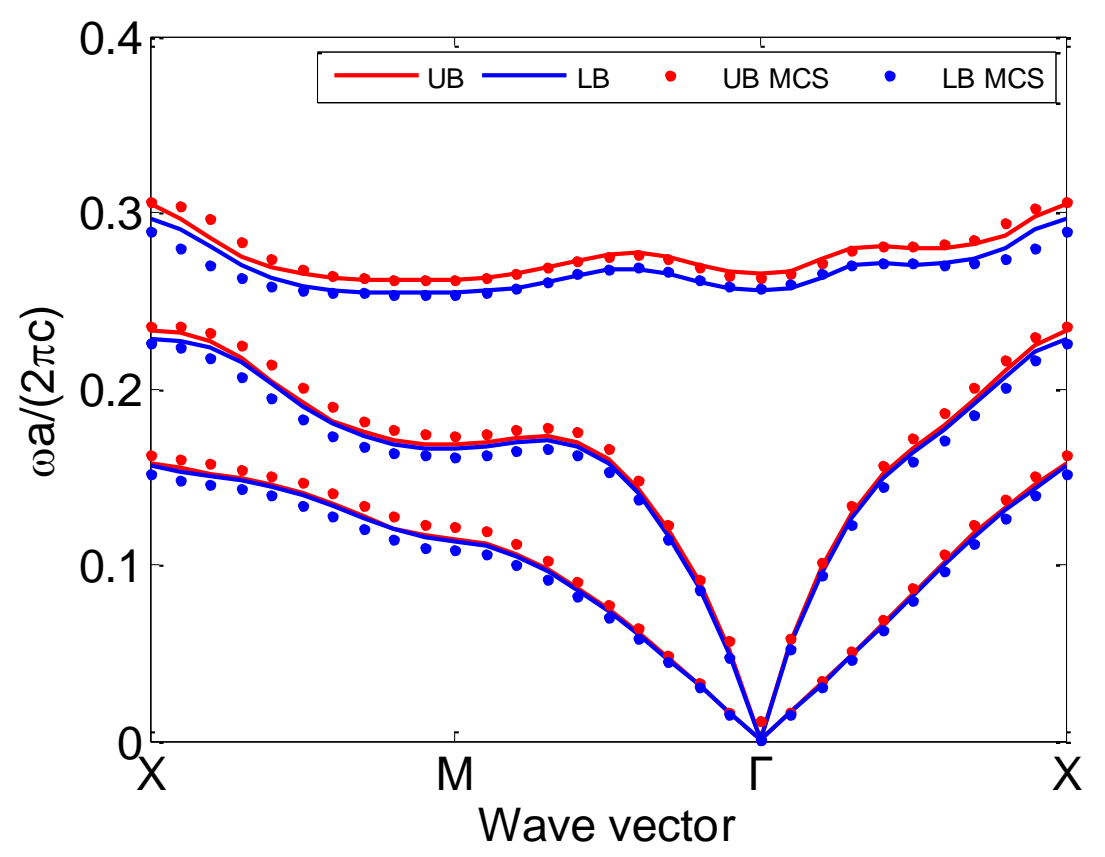

(b)

Fig. 20 The comparison of the expectations and the standard variance of the band structures computed through different methods when uncertain level is $2 \%$; (a) expectation; (b) standard variance. 
Tab. 8 Uncertainty level of the expectation of the fifth band curve

\begin{tabular}{cccccc}
\hline \multicolumn{5}{c}{ Uncertain level of the parameter $(\%)$} & \multicolumn{3}{c}{ Uncertain level $(\%)$} \\
\hline Type & Solid part $\left(\delta_{s}\right)$ & Fluid part $\left(\delta_{f}\right)$ & $\Gamma$ point & X point & M point \\
\hline 1 & 0.0 & 2.0 & 1.36 & 1.40 & 1.35 \\
2 & 0.0 & 4.0 & 2.71 & 2.79 & 2.69 \\
3 & 0.0 & 6.0 & 4.07 & 4.19 & 4.04 \\
4 & 0.0 & 8.0 & 5.43 & 5.60 & 5.40 \\
5 & 0.0 & 10.0 & 6.75 & 7.00 & 6.75 \\
6 & 2.0 & 0.0 & 0.23 & 0.62 & 0.49 \\
7 & 4.0 & 0.0 & 0.45 & 1.24 & 0.99 \\
8 & 6.0 & 0.0 & 0.68 & 1.86 & 1.49 \\
9 & 8.0 & 0.0 & 0.91 & 2.49 & 1.99 \\
10 & 10.0 & 0.0 & 1.14 & 3.1 & 2.49 \\
11 & 2.0 & 2.0 & 1.66 & 1.96 & 1.85 \\
12 & 6.0 & 6.0 & 5.01 & 5.90 & 5.55 \\
\hline
\end{tabular}

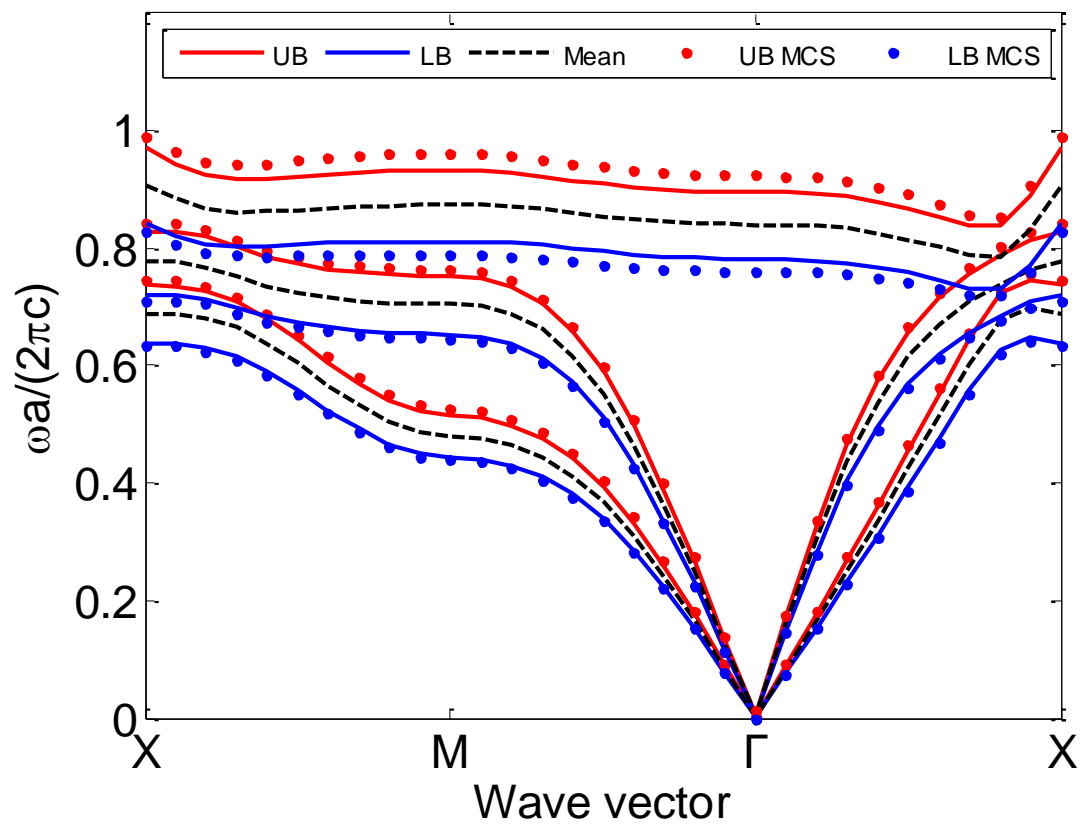

Fig. 21 The comparison of the expectations of the band structures computed through HUMR-FEM and MCS when uncertain level is $10 \%$. 


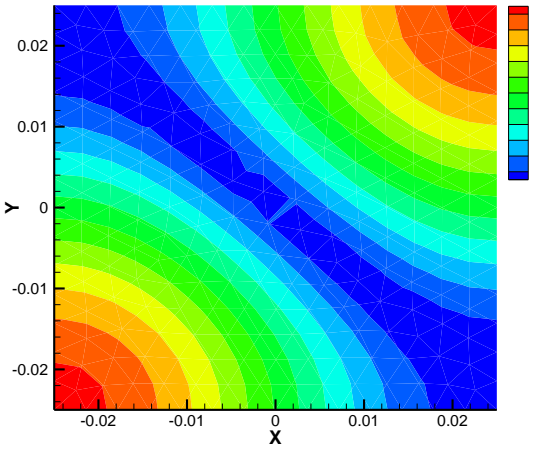

(a) Lower bound using HUMR-FEM

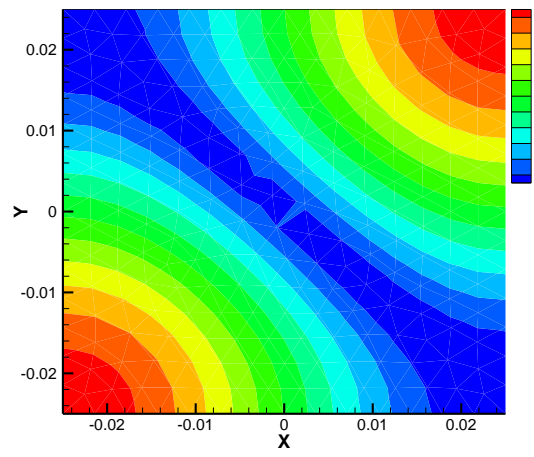

(c) Lower bound using MCS

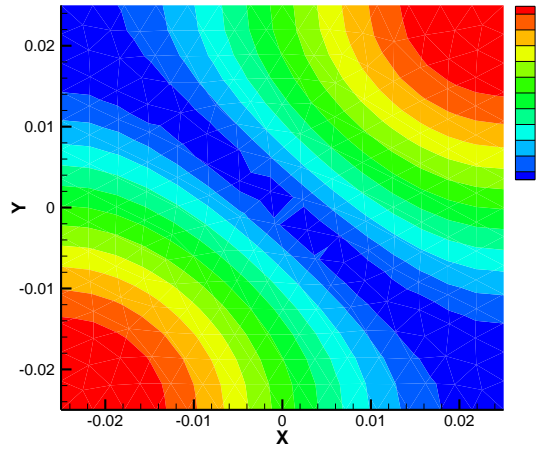

(b) Upper bound using HUMR-FEM

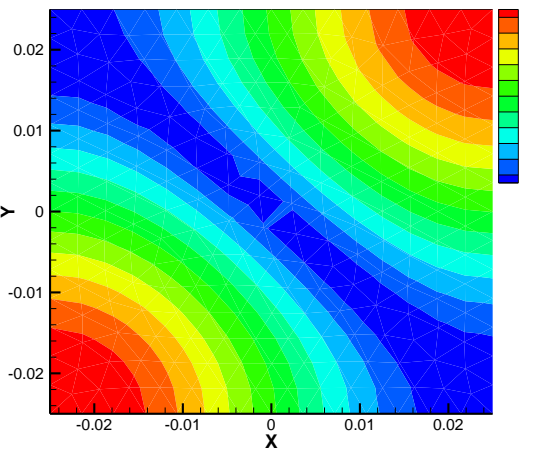

(d) Upper bound using MCS

Fig. 22 The comparison of the expectations of the eigenvectors computed through different methods when uncertain level is 2\%; (a), (b): Lower and upper bounds using HUMR-FEM; (c), (d): Lower and upper bounds using MCS.

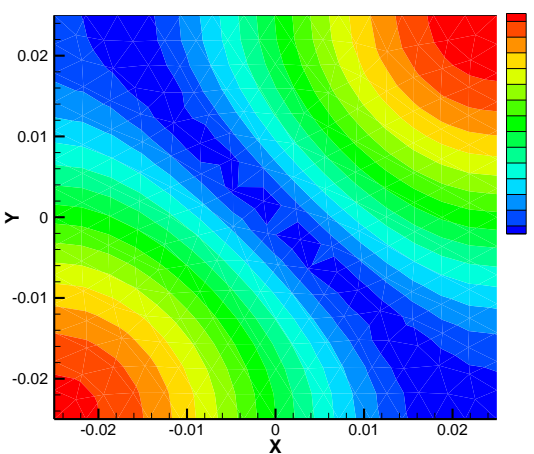

(a) Lower bound using HUMR-FEM

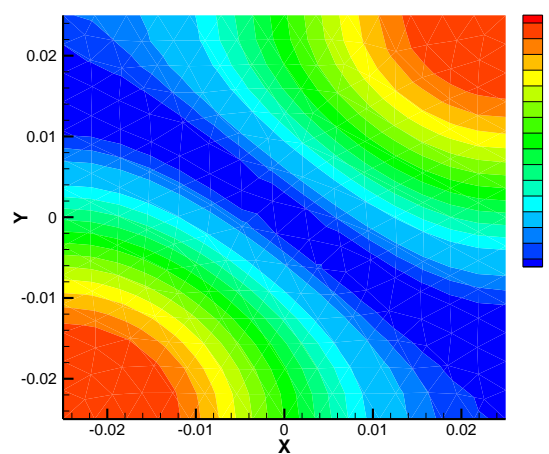

(b) Upper bound using HUMR-FEM 


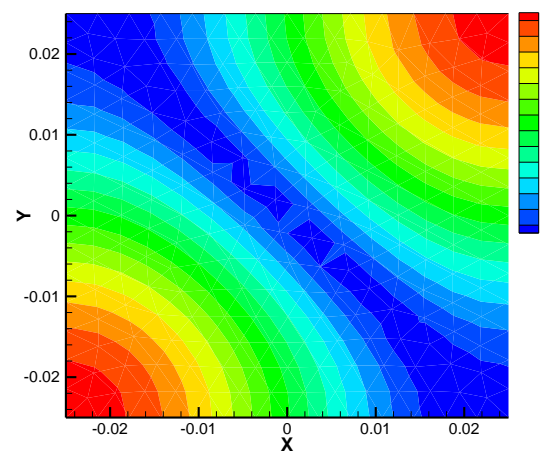

(c) Lower bound using MCS

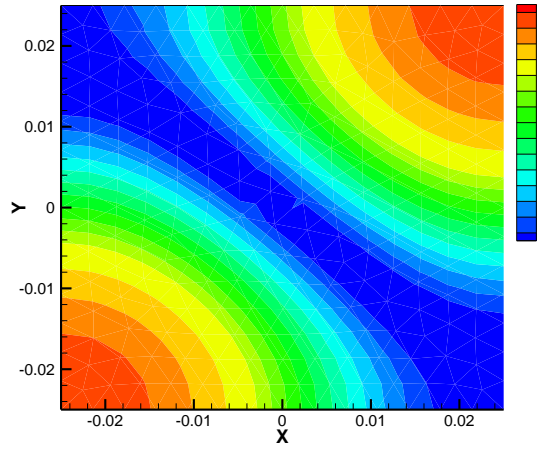

(d) Upper bound using MCS

Fig. 23 The comparison of the standard variance of the eigenvectors computed through different methods when uncertain level is 2\%; (a), (b): Lower and upper bounds using HUMR-FEM; (c), (d):

Lower and upper bounds using MCS.

Tab. 9 Computational time of using different methods (Unit: second)

\begin{tabular}{cccc}
\hline Physical responses & Statistic Property & HUMR-FEM & Monte-Carlo Method \\
\hline \multirow{2}{*}{ Band structures } & Expectation & 87.6 & 16049.6 \\
& Standard variance & 378.7 & 14146.1 \\
\hline \multirow{2}{*}{ Pressure contours } & Expectation & 1401.2 & 9573.6 \\
& Standard variance & 126.0 & 22837.6 \\
\hline
\end{tabular}

5. Conclusions

The main advantages of the present method are: 1) it provides a mass-redistributed method to minimize the dispersion error; 2) the hybrid uncertainty model integrating the advantages of the interval and the random is the first trial to investigate the uncertainty in the PCs with FSI. Results have revealed that under the influences of the hybrid uncertainties of the PCs, the band structures and pressure/displacement mode shape are subjected to the random distribution with bounded expectations and standard variances. Interestingly, this bounded interval is mainly affected by the uncertainty of the material properties from the fluid phase, while the uncertainties of the material properties from the solid phase are very small. Moreover, the uncertain levels of the physical responses increase monotonously and non-linearly as the uncertainties of the fluid domain increase linearly. In addition, the expected lower bandgap edge shifts up and the expected upper bandgap edge shifts down due to the introduction of the uncertainties, resulting in a narrower bandgap. 
It should be noted that based on the precondition of small uncertainty level $(\leq 10 \%)$ and the first-order Taylor expansion, thus this limits the method to be extended to the PCs with large uncertainty. Fortunately, this assumption is always satisfied as the practical fluid/solid or solid/fluid PCs with FSI behave a small disturbance around its expected values.

\section{Acknowledgments}

The project is supported by the National Natural Science Foundation of China (Grant No. 51322502) and Project funded by China Postdoctoral Science Foundation.

The authors also wish to thank Research Project of State Key Laboratory of Structural Analysis for Industrial Equipment (Grant No. GZ1403), the Science Fund of State Key Laboratory of Advanced Design and Manufacturing for Vehicle Body (Grant No. 51375001), and Research Project of State Key Laboratory of Mechanical Systems and Vibration MSV201613.

\section{References}

1. Liu, Z., Zhang, X., Mao, Y., Zhu, Y.Y., Yang, Z., Chan, C.T., Sheng, P.: Locally Resonant Sonic Materials. Science 289(5485), 1734 (2000). doi:10.1126/science.289.5485.1734

2. Lai, Y., Wu, Y., Sheng, P., Zhang, Z.-Q.: Hybrid elastic solids. Nature Materials 10, 620 (2011). doi:10.1038/nmat3043

https://www.nature.com/articles/nmat3043\#supplementary-information

3. Zhou, X., Hu, G.: Dynamic effective models of two-dimensional acoustic metamaterials with cylindrical inclusions. Acta Mechanica 224(6), 1233-1241 (2013). doi:10.1007/s00707-013-0870-X

4. Wu, Y., Lai, Y., Zhang, Z.-Q.: Elastic Metamaterials with Simultaneously Negative Effective Shear Modulus and Mass Density. Physical Review Letters 107(10), 105506 (2011). doi:10.1103/PhysRevLett.107.105506

5. Zhu, R., Liu, X.N., Hu, G.K., Sun, C.T., Huang, G.L.: Negative refraction of elastic waves at the deep-subwavelength scale in a single-phase metamaterial. Nature Communications 5(5), 5510 (2014).

6. Kaina, N., Lemoult, F., Fink, M., Lerosey, G.: Negative refractive index and acoustic superlens from multiple scattering in single negative metamaterials. Nature 525(7567), 77 (2015).

7. Oh, J.H., Seung, H.M., Kim, Y.Y.: Doubly negative isotropic elastic metamaterial for sub-wavelength focusing: Design and realization. Journal Of Sound And Vibration 410, 169-186 (2017). doi:10.1016/j.jsv.2017.08.027 
8. Popa, B.I., Zigoneanu, L., Cummer, S.A.: Experimental acoustic ground cloak in air. Physical Review Letters 106(25), 253901 (2011).

9. Zigoneanu, L., Popa, B.-I., Cummer, S.A.: Three-dimensional broadband omnidirectional acoustic ground cloak. Nature Materials 13, 352 (2014). doi:10.1038/nmat3901

https://www.nature.com/articles/nmat3901\#supplementary-information

10. Wu, Z.-J., Li, F.-M., Wang, Y.-Z.: Study on vibration characteristics in periodic plate structures using the spectral element method. Acta Mechanica 224(5), 1089-1101 (2013). doi:10.1007/s00707-012-0798-6

11. Zhang, G.Y., Gao, X.L., Ding, S.R.: Band gaps for wave propagation in 2-D periodic composite structures incorporating microstructure effects. Acta Mechanica 229(10), 4199-4214 (2018). doi:10.1007/s00707-018-2207-2

12. Laubie, H., Monfared, S., Radjaï, F., Pellenq, R., Ulm, F.-J.: Disorder-induced stiffness degradation of highly disordered porous materials. Journal of the Mechanics and Physics of Solids 106, 207-228 (2017). doi:https://doi.org/10.1016/j.jmps.2017.05.008

13. Chen, N., Yu, D., Xia, B., Liu, J., Ma, Z.: Interval and subinterval homogenization-based method for determining the effective elastic properties of periodic microstructure with interval parameters. International Journal of Solids and Structures 106-107, 174-182 (2017). doi:https://doi.org/10.1016/j.ijsolstr.2016.11.022

14. Li, E., He, Z.C., Hu, J.Y., Long, X.Y.: Volumetric locking issue with uncertainty in the design of locally resonant acoustic metamaterials. Computer Methods in Applied Mechanics and Engineering 324, 128-148 (2017). doi:https://doi.org/10.1016/j.cma.2017.06.005

15. He, Z.C., Hu, J.Y., Li, E.: An uncertainty model of acoustic metamaterials with random parameters. Computational Mechanics 62(5), 1023-1036 (2018). doi:10.1007/s00466-018-1548-y

16. Sukhovich, A., Jing, L., Page, J.H.: Negative refraction and focusing of ultrasound in two-dimensional phononic crystals. Physical Review B 77(1), 014301 (2008). doi:10.1103/PhysRevB.77.014301

17. Zhang, S., Yin, L., Fang, N.: Focusing Ultrasound with an Acoustic Metamaterial Network. Physical Review Letters 102(19), 194301 (2009). doi:10.1103/PhysRevLett.102.194301

18. Chen, J., Xia, B., Liu, J.: A sparse polynomial surrogate model for phononic crystals with uncertain parameters. Computer Methods in Applied Mechanics and Engineering 339, 681-703 (2018). doi:https://doi.org/10.1016/j.cma.2018.05.001

19. Wu, J., Zhang, Y., Chen, L., Luo, Z.: A Chebyshev interval method for nonlinear dynamic systems under uncertainty. Applied Mathematical Modelling 37(6), 4578-4591 (2013). doi:https://doi.org/10.1016/j.apm.2012.09.073

20. Bernard, B.P., Owens, B.A.M., Mann, B.P.: Uncertainty Propagation in the Band Gap Structure of a 1D Array of Magnetically Coupled Oscillators. Journal of Vibration and Acoustics 135(4), 041005-041005-041007 (2013). doi:10.1115/1.4023821

21. Xia, B., Yu, D., Liu, J.: Hybrid uncertain analysis of acoustic field with interval random parameters. Computer Methods in Applied Mechanics and Engineering 256, 56-69 (2013). doi:https://doi.org/10.1016/j.cma.2012.12.016

22. Elishakoff, I., Elettro, F.: Interval, ellipsoidal, and super-ellipsoidal calculi for experimental and theoretical treatment of uncertainty: Which one ought to be preferred? International Journal of $\begin{array}{lllll}\text { Solids } & \text { and } & \text { 51(7), } & \text { 1576-1586 } & \text { (2014). }\end{array}$ doi:https://doi.org/10.1016/j.ijsolstr.2014.01.010 
23. Wu, T.-T., Huang, Z.-G., Lin, S.: Surface and bulk acoustic waves in two-dimensional phononic crystal consisting of materials with general anisotropy. Physical Review B 69(9), 094301 (2004). doi:10.1103/PhysRevB.69.094301

24. Yan, Z.-Z., Wang, Y.-S.: Wavelet-based method for calculating elastic band gaps of two-dimensional phononic crystals. Physical Review B 74(22), 224303 (2006). doi:10.1103/PhysRevB.74.224303

25. Kafesaki, M., Economou, E.N.: Multiple-scattering theory for three-dimensional periodic acoustic

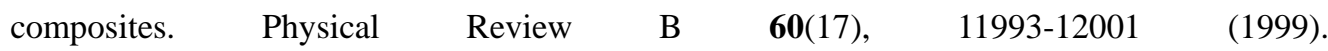
doi:10.1103/PhysRevB.60.11993

26. Shi, Z., Wang, Y., Zhang, C.: Band structure calculations of in-plane waves in two-dimensional phononic crystals based on generalized multipole technique. Applied Mathematics and Mechanics 36(5), 557-580 (2015). doi:10.1007/s10483-015-1938-7

27. Zuo, S.-L., Li, F.-M., Zhang, C.: Numerical and experimental investigations on the vibration band-gap properties of periodic rigid frame structures. Acta Mechanica 227(6), 1653-1669 (2016). doi:10.1007/s00707-016-1587-4

28. Axmann, W., Kuchment, P.: An Efficient Finite Element Method for Computing Spectra of Photonic and Acoustic Band-Gap Materials: I. Scalar Case. Journal of Computational Physics 150(2), 468-481 (1999). doi:https://doi.org/10.1006/jcph.1999.6188

29. Liu, Y., Gao, L.-t.: Explicit dynamic finite element method for band-structure calculations of 2D phononic crystals. Solid State Communications 144(3), 89-93 (2007). doi:https://doi.org/10.1016/j.ssc.2007.08.014

30. Li, F.-L., Wang, Y.-S., Zhang, C., Yu, G.-L.: Bandgap calculations of two-dimensional solid-fluid phononic crystals with the boundary element method. Wave Motion 50(3), 525-541 (2013). doi:https://doi.org/10.1016/j.wavemoti.2012.12.001

31. He, Z.C., Li, E., Wang, G., Li, G.Y., Xia, Z.: Development of an efficient algorithm to analyze the elastic wave in acoustic metamaterials. Acta Mechanica 227(10), 3015-3030 (2016). doi:10.1007/s00707-016-1664-8

32. Zheng, H., Zhang, C., Wang, Y., Chen, W., Sladek, J., Sladek, V.: A local RBF collocation method for band structure computations of 2D solid/fluid and fluid/solid phononic crystals. International Journal for Numerical Methods in Engineering 110(5), 467-500 (2017). doi:10.1002/nme.5366

33. Zheng, H., Zhang, C., Wang, Y., Sladek, J., Sladek, V.: A meshfree local RBF collocation method for anti-plane transverse elastic wave propagation analysis in 2D phononic crystals. Journal of Computational Physics 305, 997-1014 (2016). doi:https://doi.org/10.1016/j.jcp.2015.10.020

34. Li, E., He, Z.C., Wang, G., Liu, G.R.: An efficient algorithm to analyze wave propagation in fluid/solid and solid/fluid phononic crystals. Computer Methods in Applied Mechanics and Engineering 333, 421-442 (2018). doi:https://doi.org/10.1016/j.cma.2018.01.006

35. Li, F.-L., Wang, Y.-S., Zhang, C., Yu, G.-L.: Boundary element method for band gap calculations of two-dimensional solid phononic crystals. Engineering Analysis with Boundary Elements 37(2), 225-235 (2013). doi:https://doi.org/10.1016/j.enganabound.2012.10.003

36. He, Z.C., Li, E., Liu, G.R., Li, G.Y., Cheng, A.G.: A mass-redistributed finite element method (MR-FEM) for acoustic problems using triangular mesh. Journal of Computational Physics 323, 149-170 (2016). doi:https://doi.org/10.1016/j.jcp.2016.07.025 
37. Yao, L., Huang, G., Chen, H., Barnhart, M.V.: A modified smoothed finite element method (M-SFEM) for analyzing the band gap in phononic crystals. Acta Mechanica 230(6), 2279-2293 (2019). doi:10.1007/s00707-019-02396-w

38. Chadil, M.-A., Vincent, S., Estivalèzes, J.-L.: Accurate estimate of drag forces using particle-resolved direct numerical simulations. Acta Mechanica 230(2), 569-595 (2019). doi:10.1007/s00707-018-2305-1

39. Liu, G.-R., Trung, N.: Smoothed finite element methods. CRC press, (2016)

40. Wang, G., Wen, J., Liu, Y., Wen, X.: Lumped-mass method for the study of band structure in two-dimensional phononic crystals. Physical Review B 69(18), 184302 (2004). doi:10.1103/PhysRevB.69.184302

41. Xu, S., Shen, J., Zhou, S., Huang, X., Xie, Y.M.: Design of lattice structures with controlled

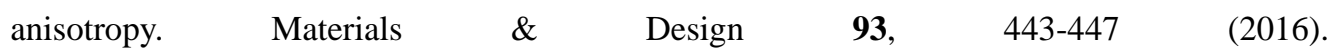
doi:https://doi.org/10.1016/j.matdes.2016.01.007

42. Cadman, J.E., Zhou, S., Chen, Y., Li, Q.: On design of multi-functional microstructural materials. Journal of Materials Science 48(1), 51-66 (2013). doi:10.1007/s10853-012-6643-4

43. Li, E., He, Z.C., Wang, G., Jong, Y.: Fundamental study of mechanism of band gap in fluid and solid/fluid phononic crystals. Advances in Engineering Software 121, 167-177 (2018). doi:https://doi.org/10.1016/j.advengsoft.2018.04.014

44. Long, X.Y., Jiang, C., Han, X.: New Method for Eigenvector-Sensitivity Analysis with Repeated Eigenvalues and Eigenvalue Derivatives. AIAA Journal 53(5), 1226-1235 (2015). doi:10.2514/1.J053362

45. Kwon, Y.W., Bang, H.: The finite element method using MATLAB (2nd ed.). CRC Press, Inc., (2000) 